\title{
A SEARCH FOR MULTI-PLANET SYSTEMS USING THE HOBBY-EBERLY TELESCOPE*
}

\author{
Robert A. Wittenmyer ${ }^{1,2}$, Michael Endl ${ }^{1}$, William D. Cochran ${ }^{1}$, Harold F. Levison $^{3}$, And Gregory W. Henry ${ }^{4}$ \\ ${ }^{1}$ McDonald Observatory, University of Texas at Austin, Austin, TX 78712, USA; rob@phys.unsw.edu.au \\ ${ }^{2}$ Department of Astrophysics, School of Physics, University of NSW, 2052, Australia \\ ${ }^{3}$ Department of Space Studies, Southwest Research Institute, Boulder, CO 80302, USA \\ ${ }^{4}$ Center of Excellence in Information Systems, Tennessee State University, 3500 John A. Merritt Blvd., Box 9501, Nashville, TN 37209, USA \\ Received 2008 December 15; accepted 2009 March 5; published 2009 April 15
}

\begin{abstract}
Extrasolar multiple-planet systems provide valuable opportunities for testing theories of planet formation and evolution. The architectures of the known multiple-planet systems demonstrate a fascinating level of diversity, which motivates the search for additional examples of such systems in order to better constrain their formation and dynamical histories. Here we describe a comprehensive investigation of 22 planetary systems in an effort to answer three questions: (1) are there additional planets? (2) where could additional planets reside in stable orbits? and (3) what limits can these observations place on such objects? We find no evidence for additional bodies in any of these systems; indeed, these new data do not support three previously announced planets (HD 20367 b: Udry et al.; HD 74156 d: Bean et al.; and 47 UMa c: Fischer et al.). The dynamical simulations show that nearly all of the 22 systems have large regions in which additional planets could exist in stable orbits. The detection-limit computations indicate that this study is sensitive to close-in Neptune-mass planets for most of the systems targeted. We conclude with a discussion on the implications of these nondetections.
\end{abstract}

Key words: methods: $N$-body simulations - planetary systems - techniques: radial velocities

Online-only material: color figures, machine readable table

\section{INTRODUCTION}

About $12 \%(N=31)$ of known planetary systems contain more than one planet. Now that radial-velocity precision at the $1-2 \mathrm{~m} \mathrm{~s}^{-1}$ level is being achieved by several planet-search programs (Butler et al. 2006; Lovis et al. 2006), Neptune-mass planets are becoming detectable. Recent discoveries of "superEarths" $\left(m \sin i \sim 10 M_{\oplus}\right)$ by the High-Accuracy Radial Velocity Planet Search (HARPS) instrument (Bouchy et al. 2009; Mayor et al. 2009; Udry et al. 2007; Bonfils et al. 2007) suggest that super-Earths may be common.

The presence of close-in giant planets ("hot Jupiters") inferred by precision radial-velocity surveys has emphasized the importance of post-formational dynamical evolution processes such as planetary migration. The core-accretion model of planetary formation (Lissauer 1995; Pollack et al. 1996) posits that rocky cores form in the outer regions of the protoplanetary disk and experience runaway gas accretion once they reach a mass of $\sim 10$ Earth masses. These giant planets then migrate inward to become hot Jupiters (Bodenheimer et al. 2000). Alternatively, the disk-instability model suggests that such planets form by direct gravitational collapse of the protoplanetary disk (Boss 1995, 1998). Multi-planet systems can be formed by this method (Boss 2003), though subsequent evolution can easily eject planets, resulting in a wide variety of system end states (Levison et al. 1998). The discovery of additional multi-planet systems will provide valuable added constraints to these two models of planet formation. Trilling et al. (1998) have proposed that gas giant planets migrating inward can overflow their Roche lobes and be stripped of their gaseous envelopes. Under

\footnotetext{
* Based on observations obtained with the Hobby-Eberly Telescope, which is a joint project of the University of Texas at Austin, the Pennsylvania State University, Stanford University, Ludwig-Maximilians-Universität München, and Georg-August-Universität Göttingen.
}

the core-accretion model of planet formation, a Neptune-mass rocky core would then remain in a close orbit, and the detection of such objects would lend support to that theory. Alternatively, the nondetection of close-in, low-mass $\left(m_{p}<15 M_{\oplus}\right)$ planets would tend to favor the disk-instability model, in which gas giant planets have no solid cores. Hence, an intensive effort to characterize the population of detectable planets around nearby stars will be extremely valuable for understanding the processes of planet formation and evolution.

The architectures of multi-planet systems can shed light on their formation and dynamical history. Chatterjee et al. (2008) performed simulations of systems with three giant planets and found that at least one planet would be ejected before the system stabilized. When two planets remained (80\% of cases), their median eccentricities were $e \sim 0$.4. Similarly, randomly generated planetary systems simulated by Jurić \& Tremaine (2008) typically retained 2-3 giant planets after $10^{8} \mathrm{yr}$. That all five planets (Fischer et al. 2008) in the 55 Cancri system have relatively low eccentricities $(e<0.2)$ suggests that systems with inactive dynamical histories (i.e., free of major perturbation events) may be able to retain several giant planets in nearly circular orbits.

The final configuration of a planetary system is dependent on the post-formation migration and dynamical interaction processes. Mandell et al. (2007) showed that the migration of a Jupiter-mass planet through a disk of planetesimals can result in the formation of an interior terrestrial-mass planet. Simulations of known multi-planet systems by Barnes \& Quinn (2004) and Barnes \& Raymond (2004) suggest that planetary systems are "packed"- that is, they contain the maximum number of planets that is dynamically possible. Barnes \& Raymond (2004) investigated the dynamically stable regions of the HD 74156 system. Those authors used the results to predict that an additional planet, between planets $\mathrm{b}$ and $\mathrm{c}$, could be present. The 
Table 1

Stellar Parameters

\begin{tabular}{|c|c|c|c|c|c|c|c|}
\hline Star & Spec. Type & $\begin{array}{c}\text { Distance } \\
(\mathrm{pc})\end{array}$ & $\begin{array}{l}\text { Mass } \\
\left(M_{\odot}\right)\end{array}$ & {$[\mathrm{Fe} / \mathrm{H}]$} & $\begin{array}{l}T_{\text {eff }} \\
(\mathrm{K})\end{array}$ & $\begin{array}{c}V \sin i \\
\left(\mathrm{~km} \mathrm{~s}^{-1}\right)\end{array}$ & $\log R_{H K}^{\prime}$ \\
\hline HD 3651 & $\mathrm{~K} 0 \mathrm{~V}$ & $11.1 \pm 0.1$ & $0.882 \pm 0.026$ & $0.24 \pm 0.03$ & $5221 \pm 44$ & 1.1 & $-4.99 \pm 0.05$ \\
\hline HD 8574 & F8 & $44.2 \pm 1.6$ & $1.122 \pm 0.022$ & $-0.03 \pm 0.03$ & $6050 \pm 44$ & 4.5 & $-4.88 \pm 0.04$ \\
\hline HD 10697 & G5IV & $32.6 \pm 0.9$ & $1.112 \pm 0.026$ & $0.17 \pm 0.03$ & $5680 \pm 44$ & 2.5 & $-5.07 \pm 0.15$ \\
\hline HD 19994 & F8V & $22.4 \pm 0.4$ & $1.365 \pm 0.042$ & $0.27 \pm 0.03$ & $6188 \pm 44$ & 8.6 & $-4.93 \pm 0.04$ \\
\hline HD 20367 & G0 & $27.1 \pm 0.8$ & $1.04 \pm 0.06^{\mathrm{a}}$ & $-0.09 \pm 0.10^{\mathrm{b}}$ & $5998 \pm 75$ & 3.0 & $-4.50 \pm 0.05$ \\
\hline HD 23596 & F8V & $52.0 \pm 2.3$ & $1.159 \pm 0.062$ & $0.33 \pm 0.03$ & $5904 \pm 44$ & 4.2 & $-4.96 \pm 0.05$ \\
\hline HD 28185 & G5 & $39.6 \pm 1.7$ & $0.98 \pm 0.05^{\mathrm{c}}$ & $0.12 \pm 0.10^{\mathrm{b}}$ & $5546 \pm 75$ & 3.0 & $-5.37 \pm 0.40$ \\
\hline HD 38529 & G4IV & $42.4 \pm 1.7$ & $1.477 \pm 0.052$ & $0.51 \pm 0.03$ & $5697 \pm 44$ & 3.9 & $-5.01 \pm 0.03$ \\
\hline HD 40979 & F8V & $33.3 \pm 0.9$ & $1.154 \pm 0.028$ & $0.15 \pm 0.03$ & $6089 \pm 44$ & 7.4 & $-4.59 \pm 0.01$ \\
\hline HD 72659 & G0V & $51.4 \pm 2.7$ & $1.068 \pm 0.022$ & $-0.02 \pm 0.03$ & $5920 \pm 44$ & 2.2 & $-5.02 \pm 0.09$ \\
\hline HD 74156 & G0 & $64.6 \pm 4.6$ & $1.238 \pm 0.044$ & $0.11 \pm 0.03$ & $6068 \pm 44$ & 4.3 & $\cdots$ \\
\hline HD 80606 & G5 & $58 \pm 20$ & $0.958 \pm 0.072$ & $0.47 \pm 0.03$ & $5573 \pm 44$ & 1.8 & $\cdots$ \\
\hline HD 89744 & F7V & $39.0 \pm 1.1$ & $1.558 \pm 0.048$ & $0.26 \pm 0.03$ & $6291 \pm 44$ & 9.5 & $-5.03 \pm 0.04$ \\
\hline $47 \mathrm{UMa}$ & G0V & $14.1 \pm 0.1$ & $1.063 \pm 0.029$ & $0.04 \pm 0.03$ & $5882 \pm 44$ & 2.8 & $-5.03 \pm 0.07$ \\
\hline HD 106252 & G0 & $37.4 \pm 1.3$ & $1.007 \pm 0.024$ & $-0.07 \pm 0.03$ & $5870 \pm 44$ & 1.9 & $-4.91 \pm 0.14$ \\
\hline HD 108874 & G5 & $68.5 \pm 5.8$ & $0.950 \pm 0.036$ & $0.19 \pm 0.03$ & $5551 \pm 44$ & 2.2 & $\cdots$ \\
\hline HD 114783 & K0 & $20.4 \pm 0.4$ & $0.853 \pm 0.034$ & $0.21 \pm 0.03$ & $5135 \pm 44$ & 0.9 & $\cdots$ \\
\hline HD 128311 & K0 & $16.6 \pm 0.3$ & $0.828 \pm 0.012$ & $0.08 \pm 0.03$ & $4965 \pm 44$ & 3.6 & $\cdots$ \\
\hline HD 130322 & $\mathrm{~K} 0 \mathrm{~V}$ & $29.8 \pm 1.3$ & $0.836 \pm 0.018$ & $-0.02 \pm 0.03$ & $5308 \pm 44$ & 1.6 & $-4.76 \pm 0.02$ \\
\hline HD 136118 & F9V & $52.3 \pm 2.3$ & $1.191 \pm 0.026$ & $-0.11 \pm 0.03$ & $6097 \pm 44$ & 7.3 & $-4.91 \pm 0.04$ \\
\hline HD $178911 \mathrm{~B}$ & G5 & $47 \pm 11$ & $1.014 \pm 0.057$ & $0.34 \pm 0.03$ & $5668 \pm 44$ & 1.9 & $-4.83 \pm 0.02$ \\
\hline HD 190228 & G5IV & $62.1 \pm 3.1$ & $1.821 \pm 0.050$ & $-0.24 \pm 0.03$ & $5348 \pm 44$ & 1.9 & $-4.98 \pm 0.02$ \\
\hline
\end{tabular}

Notes.

a Mass obtained from Holmberg et al. (2007).

${ }^{\mathrm{b}}[\mathrm{Fe} / \mathrm{H}], T_{\text {eff }}$, and $V \sin i$ obtained from Holmberg et al. (2007).

${ }^{c}$ Mass obtained from Santos et al. (2004a).

detection by Bean et al. (2008) of such an object lends support to the "packed planetary systems" hypothesis (Barnes et al. 2008), which would imply that multiple-planet systems are common. However, our own results (see Section 3) do not support this hypothesis.

A series of papers by Ida and Lin (Ida \& Lin 2004a, 2004b) predicts a paucity of planets of 10-100 Earth masses within $\sim 1$ AU (the "planet desert"). Their core-accretion simulations also predict an abundance of close-in $(a \lesssim 0.1$ AU) planets with masses below about $10 M_{\oplus}$. Ida and Lin further suggest that the distribution of planetary mass versus semimajor axis will constrain the dominant formation processes of planets. In a subsequent paper, Ida \& Lin (2008) show that the frequency of giant planets depends sensitively on the Type I migration rate, which must be slowed by a factor $C_{1} \sim$ $0.03-0.1$ in order to reproduce the distribution of detected planets.

In this work, we describe an intensive three-year radialvelocity campaign to search for additional planets in known planetary systems (Section 2). Section 3 gives the results of the orbit fits and the search for new planets, with discussion about a few of the interesting systems. Section 4 describes the dynamical simulations used to determine the regions in each system where additional planets could reside in stable orbits. The detection limits, which determine the sensitivity of this survey, are presented in Section 5. Finally, Section 6 assesses the impact of these new data and analyses on the theories of planet formation and the population-level statistics of extrasolar planets. This work thus presents a three-fold approach to the question of planetary system architecture: (1) are additional planets present in these known planetary systems? (2) where could additional objects reside in stable orbits? (3) what limits can be placed on such objects?

\section{OBSERVATIONAL DATA}

22 targets were chosen for this project from the list of $\sim 150$ planet hosts known in 2004 September. A majority of the observational data were obtained at McDonald Observatory with the 9.2 m Hobby-Eberly Telescope (HET; Ramsey et al. 1998) using its High Resolution Spectrograph (HRS; Tull 1998). The targets were selected according to the following criteria: (1) HET observability, with declination between $-11^{\circ}$ and $+72^{\circ},(2)$ either a long-period $(P \gtrsim 1 \mathrm{yr})$ planet such that inner planets may be dynamically stable, or a very short-period ( $P \lesssim 10$ days) hot Jupiter which would allow for previously undetected outer planets, and (3) the orbital solution for the known planet in each system has RV residuals of $10-20 \mathrm{~m} \mathrm{~s}^{-1}$, so that an additional planet may be present but undetected. The targets and their stellar parameters are listed in Table 1. Except where noted, masses are obtained from Takeda et al. (2007), $[\mathrm{Fe} / \mathrm{H}], T_{\mathrm{eff}}$, and $V \sin i$ from Valenti \& Fischer (2005), and the chromospheric emission ratio $\log R_{H K}^{\prime}$ (Noyes et al. 1984) computed from measurements of the Ca II S-index obtained with the $2.7 \mathrm{~m}$ telescope using the techniques developed by Paulson et al. (2002). The uncertainties on the stellar masses given in Takeda et al. (2007) are asymmetric about the central value; for the purposes of Table 1 and the determination of planetary parameters, the adopted stellar mass uncertainty was taken to be the larger of the two.

All of the HET observations for this program were performed at a spectral resolution of 60,000 , with the 316 lines $\mathrm{mm}^{-1}$ crossdisperser and a central wavelength of $5936 \AA$. An iodine cell temperature controlled at $70^{\circ} \mathrm{C}$ was used as the velocity metric (Marcy \& Butler 1992). This setup, identical to that used for the ongoing planet-search program (Cochran et al. 2004; Endl et al. 2008), places the iodine region ( 5000-6000 $)$ almost entirely 
Table 2

Summary of Published Radial-Velocity Data

\begin{tabular}{|c|c|c|c|c|}
\hline Star & Reference & $N$ & $\begin{array}{c}\langle\sigma\rangle \\
\left(\mathrm{m} \mathrm{s}^{-1}\right)\end{array}$ & $\begin{array}{l}\text { rms about fit } \\
\left(\mathrm{m} \mathrm{s}^{-1}\right)\end{array}$ \\
\hline HD 3651 & Butler et al. (2006) & 163 & 3.4 & 6.6 \\
\hline HD 8574 & Perrier et al. (2003) & 41 & 10.3 & 13.1 \\
\hline HD 8574 & Butler et al. (2006) & 26 & 10.4 & 23.0 \\
\hline HD 10697 & Butler et al. (2006) & 59 & 2.7 & 6.8 \\
\hline HD 19994 & Mayor et al. (2004) & 48 & 6.7 & 8.1 \\
\hline HD 23596 & Perrier et al. (2003) & 39 & 9.1 & 9.2 \\
\hline HD 28185 & Santos et al. (2001) & 40 & 6.5 & 10.0 \\
\hline HD 38529 & Butler et al. (2006) & 162 & 5.3 & 13 \\
\hline HD 40979 & Butler et al. (2006) & 65 & 9.1 & 23 \\
\hline HD 72659 & Butler et al. (2006) & 32 & 3.2 & 4.2 \\
\hline HD 74156 & Naef et al. (2004) & 95 & 10.8 & 10.6 \\
\hline HD 80606 & Naef et al. (2001) & 61 & 13.7 & 17.7 \\
\hline HD 89744 & Butler et al. (2006) & 50 & 11.2 & 16.0 \\
\hline $47 \mathrm{UMa}$ & Fischer et al. (2002) & 91 & 5.7 & 7.4 \\
\hline $47 \mathrm{UMa}$ & Naef et al. (2004) & 44 & 7.3 & 7.4 \\
\hline HD 106252 & Perrier et al. (2003) & 40 & 10.7 & 10.5 \\
\hline HD 106252 & Butler et al. (2006) & 15 & 11.4 & 9.1 \\
\hline HD 108874 & Vogt et al. (2005) & 49 & 3.4 & 3.7 \\
\hline HD 114783 & Butler et al. (2006) & 54 & 2.7 & 4.7 \\
\hline HD 128311 & Vogt et al. (2005) & 76 & 3.3 & 18.0 \\
\hline HD 130322 & Udry et al. (2000) & 118 & 12.4 & 16.1 \\
\hline HD 130322 & Butler et al. (2006) & 12 & 2.7 & 11.0 \\
\hline HD 136118 & Butler et al. (2006) & 37 & 16.1 & 22.0 \\
\hline HD $178911 \mathrm{~B}$ & Zucker et al. (2002) & 51 & 10.4 & 11.0 \\
\hline HD $178911 \mathrm{~B}$ & Butler et al. (2006) & 14 & 2.7 & 7.7 \\
\hline HD 190228 & Perrier et al. (2003) & 51 & 8.7 & 8.0 \\
\hline
\end{tabular}

onto the blue CCD, which is cosmetically superior to the red CCD. For each target, an iodine-free template spectrum was obtained near the beginning of the first season in which it was observable. We determined precise radial velocities following the general recipe outlined by Butler et al. (1996), using an advanced version of our own code "Austral" (Endl et al. 2000).

We observed each target with the HET in queue mode using a random observing interval of 2-10 days between visits. Each visit consisted of one spectrum, except for seven bright targets (HD 3651, HD 19994, HD 38529, HD 74156, 47 UMa, HD 128311, and HD 136118) for which three consecutive spectra were obtained in each visit. HET data consisting of multiple exposures per visit were binned using the weighted mean value of the velocities in each visit. We adopted the quadrature sum of the rms about the mean and the mean internal error as the error bar of each binned point. This procedure was done for HD 3651, HD 19994, HD 38529, HD 74156, $47 \mathrm{UMa}$, HD 128311, and HD 136118. Targets were observed with the HET from 2004 December through 2007 November. During the three years of this study, supplemental observations were also made using the $2.7 \mathrm{~m}$ Harlan J. Smith telescope at McDonald Observatory. All available published radial-velocity data were also gathered from the literature for the purpose of fitting orbits to the known planets. Those data are summarized in Table 2. All radial-velocity data obtained from McDonald Observatory are given in Table 7 .

\section{REFINED PLANETARY SYSTEM PARAMETERS}

\subsection{Orbit-Fitting Results}

Available published data were combined with velocities from the HET and the $2.7 \mathrm{~m}$ to fit Keplerian orbits using GaussFit (Jefferys et al. 1987), which is a generalized least-squares program used here to solve a Keplerian radial-velocity orbit model. The GaussFit model has the ability to allow the offsets between data sets to be a free parameter. This is important because the radial velocities cited in published works, and those computed from HET and $2.7 \mathrm{~m}$ data, are not absolute radial velocities, but rather are measured relative to the iodinefree stellar template. The Geneva planet-search group, however, makes use of a simultaneous thorium-argon calibration rather than an iodine absorption cell (Baranne et al. 1996). Each data set thus has an arbitrary zero-point offset which must be accounted for in the orbit-fitting procedure.

The best-fit Keplerian orbital solutions and planetary parameters are shown in Table 3. A summary of the fit results for each individual data set is given in Table 4. In computing the planetary minimum mass $M \sin i$ and semimajor axis $a$, the stellar masses listed in Table 1 were used. The addition of a large amount of new data and the use of multiple independent data sets in fitting Keplerian orbits have generally improved the precision of the derived planetary parameters by a factor of 2-4 over the published results summarized in the Catalog of Nearby Exoplanets (Butler et al. 2006). In particular, the precision of the orbital periods have been improved by the addition of new data, due to the increased number of orbits now observed. Our parameters generally agree within $2 \sigma$ of previously published estimates. In this section, we highlight interesting results from the combined fits.

For each object, we searched for periodic signals in the residuals to the known planet's orbit using a Lomb-Scargle periodogram (Lomb 1976; Scargle 1982). To assess the statistical significance of those periods, the false alarm probabilities (FAPs) were calculated using the bootstrap randomization method detailed by Kürster et al. (1997). The bootstrap method randomly shuffles the velocity observations while keeping the times of observation fixed. The periodogram of this shuffled data set is then computed and its highest peak recorded. In this way, we can determine the probability that a periodogram peak of a given power level will arise by chance, without making any assumptions about the error distribution of the data. All bootstrap FAP estimates result from 10,000 such realizations. Those results are shown in Table 5.

HD 20367. A planet orbiting HD 20367 was first announced in a conference proceedings (Udry et al. 2003), but has not yet appeared in a refereed journal. The Geneva planet-search group Web site ${ }^{5}$ lists the planet's period as 469.5 days, with an eccentricity of 0.32 and $M \sin i=1.17 M_{\mathrm{Jup}} .81$ observations of HD 20367 were obtained with the HET over three observing seasons, as well as 19 observations from the $2.7 \mathrm{~m}$, but period searches of these data give no indication of such a signal.

Figure 1 shows the radial-velocity data from HET and the $2.7 \mathrm{~m}$ telescopes, and the periodogram of those data. The Geneva group's solution has been overplotted. The highest peak, at 5.58 days, has a bootstrap FAP of $8.5 \%$. The dominant periodicity of 5.58 days, which was evident early in the observation campaign, prompted a photometric investigation to search for transits and to rule out stellar rotation. We obtained 132 observations of HD 20367 from 2006 September to 2007 January with the T10 $0.8 \mathrm{~m}$ automated photometric telescope (APT) at Fairborn Observatory in southern Arizona. The T10 APT and its precision photometer are very similar to the T8 APT described in Henry (1999). The precision of a single observation is typically around $0.001 \mathrm{mag}$. The results indicate a stellar rotation

\footnotetext{
5 http://obswww.unige.ch/ udry/planet/hd20367.html
} 
Table 3

Keplerian Orbital Solutions

\begin{tabular}{|c|c|c|c|c|c|c|c|c|c|}
\hline Planet & $\begin{array}{l}\text { Period } \\
\text { (Days) }\end{array}$ & $\begin{array}{c}T_{0} \\
(\mathrm{JD}-2400000) \\
\end{array}$ & $e$ & $\begin{array}{c}\omega \\
(\mathrm{deg})\end{array}$ & $\begin{array}{c}K \\
\left(\mathrm{~m} \mathrm{~s}^{-1}\right) \\
\end{array}$ & $\begin{array}{c}M \sin i \\
\left(M_{\text {Jup }}\right)\end{array}$ & $\begin{array}{c}a \\
(\mathrm{AU})\end{array}$ & $\chi^{2} v$ & $\begin{array}{c}\mathrm{rms} \\
\mathrm{m} \mathrm{s}^{-1}\end{array}$ \\
\hline HD $3651 \mathrm{~b}$ & $62.218 \pm 0.015$ & $53932.6 \pm 0.6$ & $0.596 \pm 0.036$ & $242.5 \pm 4.5$ & $15.9 \pm 0.7$ & $0.229 \pm 0.008$ & $0.295 \pm 0.003$ & 3.82 & 6.3 \\
\hline HD 8574 b & $227.0 \pm 0.2$ & $53981.0 \pm 3.2$ & $0.297 \pm 0.026$ & $26.6 \pm 5.4$ & $58.3 \pm 1.8$ & $1.80 \pm 0.06$ & $0.757 \pm 0.005$ & 2.21 & 14.2 \\
\hline HD 10697 b & $1075.2 \pm 1.5$ & $51480 \pm 18$ & $0.099 \pm 0.007$ & $111.2 \pm 6.3$ & $115.4 \pm 1.1$ & $6.21 \pm 0.15$ & $2.131 \pm 0.018$ & 3.39 & 8.1 \\
\hline HD 19994 b & $466.2 \pm 1.7$ & $53757 \pm 72$ & $0.063 \pm 0.062$ & $346 \pm 55$ & $29.3 \pm 2.1$ & $1.37 \pm 0.12$ & $1.305 \pm 0.016$ & 5.27 & 14.0 \\
\hline HD 23596 b & $1561 \pm 12$ & $53163 \pm 22$ & $0.266 \pm 0.014$ & $272.6 \pm 3.3$ & $127.0 \pm 2.0$ & $7.71 \pm 0.39$ & $2.772 \pm 0.062$ & 0.88 & 8.7 \\
\hline HD $28185 b$ & $385.9 \pm 0.6$ & $53793.6 \pm 8.8$ & $0.092 \pm 0.019$ & $351.9 \pm 8.2$ & $158.8 \pm 4.2$ & $5.59 \pm 0.33$ & $1.032 \pm 0.019$ & 2.28 & 9.5 \\
\hline HD 38529 b & $14.3098 \pm 0.0005$ & $54012.64 \pm 0.16$ & $0.257 \pm 0.015$ & $92.5 \pm 3.9$ & $56.1 \pm 0.9$ & $0.839 \pm 0.030$ & $0.131 \pm 0.002$ & 6.32 & 11.8 \\
\hline HD $38529 \mathrm{c}$ & $2140.2 \pm 5.7$ & $52256.4 \pm 6.4$ & $0.341 \pm 0.005$ & $17.8 \pm 1.2$ & $173.2 \pm 1.2$ & $13.38 \pm 0.39$ & $3.712 \pm 0.048$ & 6.32 & 11.8 \\
\hline HD $40979 \mathrm{~b}$ & $264.15 \pm 0.23$ & $53919.0 \pm 2.7$ & $0.252 \pm 0.014$ & $323.4 \pm 4.1$ & $119.4 \pm 2.2$ & $4.01 \pm 0.13$ & $0.846 \pm 0.007$ & 4.44 & 20.3 \\
\hline HD 72659 b & $3383 \pm 100$ & $51572 \pm 52$ & $0.271 \pm 0.022$ & $241 \pm 8$ & $42.4 \pm 1.1$ & $3.15 \pm 0.14$ & $4.511 \pm 0.114$ & 1.00 & 6.6 \\
\hline HD $74156 b^{a}$ & $51.645 \pm 0.003$ & $53788.59 \pm 0.09$ & $0.627 \pm 0.009$ & $176.5 \pm 1.2$ & $109.6 \pm 2.3$ & $1.80 \pm 0.06$ & $0.292 \pm 0.004$ & 1.60 & 11.5 \\
\hline HD $74156 c$ & $2473 \pm 13$ & $53415 \pm 13$ & $0.432 \pm 0.013$ & $258.6 \pm 2.7$ & $116.5 \pm 3.3$ & $8.06 \pm 0.37$ & $3.850 \pm 0.054$ & 1.60 & 11.5 \\
\hline HD 80606 b & $111.429 \pm 0.001$ & $53421.923 \pm 0.004$ & $0.9324 \pm 0.0006$ & $300.4 \pm 0.3$ & $470.6 \pm 1.8$ & $3.91 \pm 0.19$ & $0.447 \pm 0.011$ & 1.41 & 13.3 \\
\hline HD 89744 b & $256.78 \pm 0.05$ & $51505.5 \pm 0.4$ & $0.673 \pm 0.007$ & $195.1 \pm 1.0$ & $271.6 \pm 4.0$ & $8.44 \pm 0.23$ & $0.918 \pm 0.010$ & 2.58 & 15.2 \\
\hline $47 \mathrm{UMa} \mathrm{b}^{\mathrm{b}}$ & $1076.6 \pm 2.3$ & $49222 \pm 347$ & $0.012 \pm 0.023$ & $147 \pm 117$ & $46.6 \pm 1.1$ & $2.45 \pm 0.10$ & $2.100 \pm 0.022$ & 3.61 & 10.2 \\
\hline HD $106252 \mathrm{~b}$ & $1531.0 \pm 4.7$ & $53397.5 \pm 4.7$ & $0.482 \pm 0.011$ & $292.8 \pm 1.8$ & $138.8 \pm 2.0$ & $6.92 \pm 0.16$ & $2.611 \pm 0.026$ & 1.42 & 12.2 \\
\hline HD $108874 b$ & $395.8 \pm 0.6$ & $54069 \pm 17$ & $0.082 \pm 0.021$ & $232 \pm 10$ & $37.0 \pm 0.8$ & $1.29 \pm 0$ & $1.038 \pm 0.014$ & 0.88 & 4.1 \\
\hline HD $108874 \mathrm{c}$ & $1624 \pm 23$ & $52839 \pm 44$ & $0.239 \pm 0.031$ & $27 \pm 10$ & $18.2 \pm 0.7$ & $0.99 \pm 0.06$ & $2.659 \pm 0.060$ & 0.88 & 4.1 \\
\hline HD $114783 b$ & $493.7 \pm 1.8$ & $53806 \pm 14$ & $0.144 \pm 0.032$ & $86 \pm 11$ & $31.9 \pm 0.9$ & $1.10 \pm 0.06$ & $1.160 \pm 0.019$ & 4.91 & 6.3 \\
\hline HD $128311 b$ & $454.2 \pm 1.6$ & $53835 \pm 11$ & $0.345 \pm 0.049$ & $63 \pm 16$ & $46.5 \pm 4.5$ & $1.45 \pm 0.13$ & $1.086 \pm 0.008$ & 21.38 & 16.9 \\
\hline HD $128311 \mathrm{c}$ & $923.8 \pm 5.3$ & $56987 \pm 41$ & $0.230 \pm 0.058$ & $28 \pm 15$ & $78.8 \pm 2.6$ & $3.24 \pm 0.10$ & $1.745 \pm 0.017$ & 21.38 & 16.9 \\
\hline HD $130322 b^{c}$ & $10.7085 \pm 0.0003$ & $53995.0 \pm 2.3$ & $0.011 \pm 0.020$ & $145 \pm 77$ & $108.3 \pm 2.0$ & $1.04 \pm 0.03$ & $0.0896 \pm 0.0006$ & 4.29 & 8.9 \\
\hline HD $136118 b$ & $1187.3 \pm 2.4$ & $52999.5 \pm 5.3$ & $0.338 \pm 0.015$ & $319.9 \pm 2.1$ & $210.7 \pm 2.5$ & $11.60 \pm 0.25$ & $2.333 \pm 0.020$ & 1.82 & 16.5 \\
\hline HD $178911 \mathrm{~B} \mathrm{~b}$ & $71.484 \pm 0.002$ & $53808.1 \pm 0.3$ & $0.114 \pm 0.003$ & $168.2 \pm 1.6$ & $343.3 \pm 1.0$ & $7.03 \pm 0.28$ & $0.339 \pm 0.006$ & 1.80 & 9.1 \\
\hline HD 190228 b & $1136.1 \pm 9.9$ & $53522 \pm 12$ & $0.531 \pm 0.028$ & $101.2 \pm 2.1$ & $91.4 \pm 3.0$ & $5.93 \pm 0.20$ & $2.604 \pm 0.032$ & 0.78 & 7.4 \\
\hline
\end{tabular}

Notes.

${ }^{a}$ Results from two-planet fit.

${ }^{b}$ Results from one-planet fit.

${ }^{c}$ Results for HD 130322 exclude data from Udry et al. (2000).

period of $5.50 \pm 0.02$ days, with a photometric amplitude of $0.0055 \pm 0.0003 \mathrm{mag}$ (Figure 2). From these observations, we conclude that the 5.6-day radial-velocity periodicity is caused by starspots rotating into and out of view. This is consistent with the estimate of $P_{\text {rot }}=6$ days reported by Wright et al. (2004), and the high level of chromospheric activity for this star $\left(\log R_{H K}^{\prime}=-4.50\right)$. The literature contains conflicting age estimates for HD 20367: Holmberg et al. (2007) estimate an age of $4.4_{-2.1}^{+1.6} \mathrm{Gyr}$, whereas Wright et al. (2004) report an age of $0.9 \mathrm{Gyr}$. Based on the rapid rotation rate, and the high level of chromospheric emission, the younger age estimate is favored.

The lack of any Keplerian signal in the 100 observations presented here leads us to conclude that there is not convincing evidence for the existence of HD $20367 \mathrm{~b}$.

HD 74156. For HD 74156, we fit the two planets at 51 and 2473 days using ELODIE and CORALIE data from Naef et al. (2004), and 82 independent HET visits. This system warrants closer scrutiny in light of the report by Bean et al. (2008) of a third planet, with a period of 346 days and a radial-velocity semiamplitude $K=10.5 \mathrm{~m} \mathrm{~s}^{-1}$. That result was obtained using the same HET spectra as considered in this work, but velocities were derived using an independent method described in Bean et al. (2007). Here, we further investigate the possibility of an additional planet in the HD 74156 system. Applying our orbit-fitting methods as described above to the velocities for HD 74156 given in Bean et al. (2008), a periodogram peak is evident near 346 days, and we obtain a three-planet Keplerian orbit fit which is consistent with that of Bean et al. (2008). This indicates that the fitting method used here is not responsible for our nondetection of HD $74156 \mathrm{~d}$.
It is possible that the HET velocities derived by Bean et al. (2008) are of superior quality to those presented here. However, the rms of the HET data about a two-planet fit reported by Bean et al. (2008) is $8.5 \mathrm{~m} \mathrm{~s}^{-1}$, whereas we obtain an rms of $8.3 \mathrm{~m} \mathrm{~s}^{-1}$ for those data. These results suggest that there is no significant difference in quality between the two extant sets of HET velocities for HD 74156. The uncertainties quoted by Bean et al. (2008) are generally smaller than ours by a factor of $2-3$. We repeated the fitting procedure, reducing the HET uncertainties by a factor of 2 and 3 , but there was no significant change in the residuals: no signal is evident at periods near 346 days.

Since the total rms scatter about our two-planet fit is $11.5 \mathrm{~m}$ $\mathrm{s}^{-1}$, and the semiamplitude of planet $\mathrm{d}$ is $K=10.5 \mathrm{~m} \mathrm{~s}^{-1}$, it is possible that a third planetary signal may have been lost in the noise. To test this possibility, we performed the following Monte Carlo simulations. From each of the two data sets considered in the fits described here, we generated 1000 simulated sets of velocities consisting of three Keplerian signals plus a Gaussian noise term. This noise was equivalent to the mean uncertainty of each data set (ELODIE + CORALIE: $10.8 \mathrm{~m} \mathrm{~s}^{-1}$, HET: $8.3 \mathrm{~m} \mathrm{~s}^{-1}$ ) added in quadrature to a stellar jitter of $4 \mathrm{~m} \mathrm{~s}^{-1}$ (the jitter estimate used in Bean et al. 2008). The parameters of the three simulated planets were those from Bean et al. (2008). These simulated data sets retained the times of observation and the error bars of the originals. We then fit the simulated data with a two-planet model exactly as described above, and examined the residuals of the two-planet fit by the periodogram method, to determine whether the signal of planet $d$ was recovered. The criteria for recovery were that the period of the second planet had to be detected correctly and with an FAP of less than $0.1 \%$. This 
Table 4

Summary of Radial-Velocity Data

\begin{tabular}{|c|c|c|c|c|}
\hline Star & $N$ & rms About Fit $\left(\mathrm{m} \mathrm{s}^{-1}\right)$ & $\Delta T$ (Days) & Source \\
\hline HD 3651 & 163 & 6.5 & & Butler et al. (2006) \\
\hline HD 3651 & 35 & 5.1 & & $\mathrm{HET}^{\mathrm{a}}$ \\
\hline HD 3651 & 4 & 9.3 & & $2.7 \mathrm{~m}^{\mathrm{b}}$ \\
\hline HD 3651 (total) & 202 & 6.3 & 7376 & \\
\hline HD 8574 & 41 & 14.8 & & Perrier et al. (2003) \\
\hline HD 8574 & 44 & 8.7 & & HET \\
\hline HD 8574 & 16 & 13.4 & & $2.7 \mathrm{~m}$ \\
\hline HD 8574 & 26 & 20.7 & & Butler et al. (2006) \\
\hline HD 8574 (total) & 128 & 14.2 & 3609 & \\
\hline HD 10697 & 59 & 6.5 & & Butler et al. (2006) \\
\hline HD 10697 & 32 & 8.8 & & $2.7 \mathrm{~m}$ \\
\hline HD 10697 & 40 & 9.7 & & HET \\
\hline HD 10697 (total) & 131 & 8.1 & 4057 & \\
\hline HD 19994 & 48 & 14.8 & & Mayor et al. (2004) \\
\hline HD 19994 & 56 & 12.5 & & HET \\
\hline HD 19994 & 12 & 18.5 & & $2.7 \mathrm{~m}$ \\
\hline HD 19994 (total) & 116 & 14.0 & 3367 & \\
\hline HD $20367^{c}$ & 81 & 12.9 & & HET \\
\hline HD 20367 & 19 & 10.5 & & $2.7 \mathrm{~m}$ \\
\hline HD 20367 (total) & 100 & 12.4 & 974 & \\
\hline HD 23596 & 39 & 9.4 & & Perrier et al. (2003) \\
\hline HD 23596 & 63 & 8.5 & & HET \\
\hline HD 23596 & 6 & 5.8 & & $2.7 \mathrm{~m}$ \\
\hline HD 23596 (total) & 108 & 8.7 & 3603 & \\
\hline HD 28185 & 40 & 10.4 & & Santos et al. (2001) \\
\hline HD 28185 & 34 & 8.5 & & HET \\
\hline HD 28185 (total) & 74 & 9.5 & 2971 & \\
\hline HD 38529 & 162 & 13.0 & & Butler et al. (2006) \\
\hline HD 38529 & 73 & 8.9 & & HET \\
\hline HD 38529 & 7 & 9.2 & & $2.7 \mathrm{~m}$ \\
\hline HD 38529 (total) & 242 & 11.8 & 3745 & \\
\hline HD 40979 & 65 & 22.8 & & Butler et al. (2006) \\
\hline HD 40979 & 91 & 18.9 & & HET \\
\hline HD 40979 & 4 & 9.6 & & $2.7 \mathrm{~m}$ \\
\hline HD 40979 (total) & 160 & 20.3 & 3588 & \\
\hline HD 72659 & 32 & 4.1 & & Butler et al. (2006) \\
\hline HD 72659 & 53 & 7.8 & & HET \\
\hline HD 72659 (total) & 85 & 6.6 & 3593 & \\
\hline HD 74156 & 95 & 13.8 & & Naef et al. (2004) \\
\hline HD 74156 & 82 & 8.3 & & HET \\
\hline HD 74156 (total) & 177 & 11.5 & 3408 & \\
\hline HD 80606 & 61 & 18.6 & & Naef et al. (2001) \\
\hline HD 80606 & 23 & 6.1 & & HET \\
\hline HD 80606 & 46 & 5.3 & & Butler et al. (2006) \\
\hline HD 80606 (total) & 130 & 13.3 & 2893 & \\
\hline HD 89744 & 50 & 16.2 & & Butler et al. (2006) \\
\hline HD 89744 & 33 & 12.9 & & HET \\
\hline HD 89744 & 9 & 19.0 & & $2.7 \mathrm{~m}$ \\
\hline HD 89744 (total) & 92 & 15.2 & 2943 & \\
\hline $47 \mathrm{UMa}$ & 91 & 11.1 & & Fischer et al. (2002) \\
\hline $47 \mathrm{UMa}$ & 44 & 11.8 & & Naef et al. (2004) \\
\hline $47 \mathrm{UMa}$ & 43 & 11.4 & & $2.7 \mathrm{~m}$ \\
\hline $47 \mathrm{UMa}$ & 77 & 7.0 & & HET \\
\hline 47 UMa (total) & 255 & 10.2 & 7673 & \\
\hline HD 106252 & 40 & 14.8 & & Perrier et al. (2003) \\
\hline HD 106252 & 43 & 8.2 & & HET \\
\hline HD 106252 & 15 & 12.2 & & Butler et al. (2006) \\
\hline HD 106252 & 12 & 16.1 & & $2.7 \mathrm{~m}$ \\
\hline HD 106252 (total) & 110 & 12.2 & 3682 & \\
\hline HD 108874 & 49 & 3.4 & & Vogt et al. (2005) \\
\hline HD 108874 & 40 & 4.8 & & HET \\
\hline HD 108874 (total) & 89 & 4.1 & 2850 & \\
\hline HD 114783 & 54 & 6.6 & & Butler et al. (2006) \\
\hline HD 114783 & 34 & 5.8 & & HET \\
\hline HD 114783 (total) & 88 & 6.3 & 3208 & \\
\hline HD 128311 & 76 & 15.8 & & Vogt et al. (2005) \\
\hline
\end{tabular}

Table 4

Continued

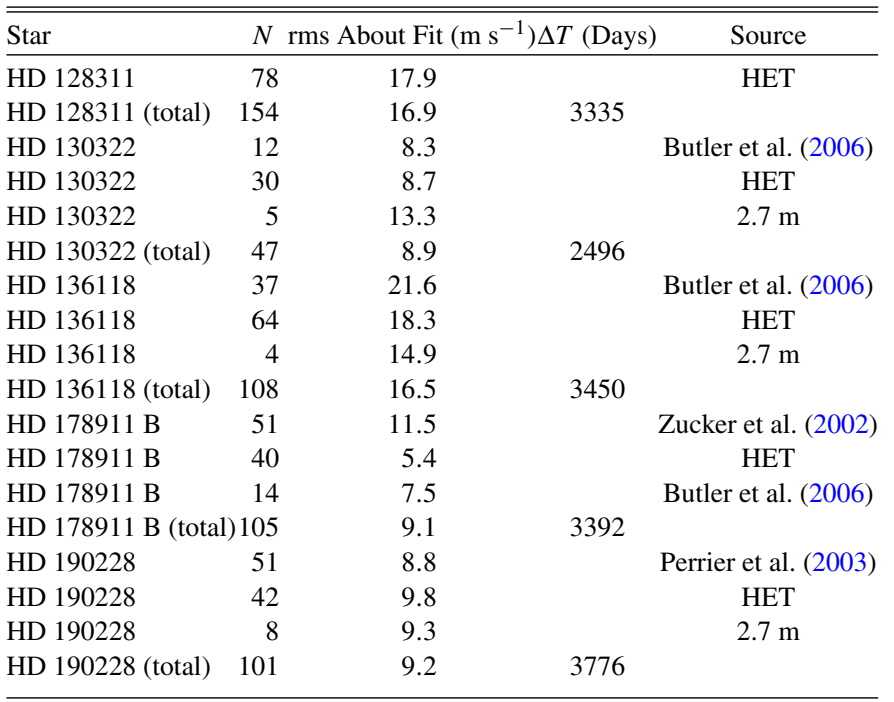

Notes.

a $9.2 \mathrm{~m}$ Hobby-Eberly Telescope.

b McDonald Observatory 2.7 m Harlan J. Smith Telescope.

${ }^{\mathrm{c}}$ No planet was fitted.

Table 5

Results of Periodogram Analysis

\begin{tabular}{lrc}
\hline \hline Star & Period (Days) & FAP \\
\hline HD 3651 & 44.17 & 0.707 \\
HD 8574 & 2272.73 & 0.687 \\
HD 10697 & 26.68 & 0.028 \\
HD 19994 & 54.88 & 0.399 \\
HD 20367 & 5.58 & 0.085 \\
HD 23596 & 25.13 & 0.141 \\
HD 28185 & 4.76 & 0.224 \\
HD 38529 & 294.12 & 0.023 \\
HD 40979 & 2.26 & 0.795 \\
HD 72659 & 6.99 & 0.758 \\
HD 74156 & 80.39 & 0.035 \\
HD 80606 & 357.14 & 0.616 \\
HD 89744 & 23.27 & 0.075 \\
47 UMa & 2380.95 & 0.045 \\
47 UMa & 2.91 & 0.341 \\
HD 106252 & 322.58 & 0.126 \\
HD 108874 & 12.39 & 0.857 \\
HD 114783 & 8.44 & 0.925 \\
HD 128311 & 11.21 & $<0.0001$ \\
HD 130322 & 438.60 & 0.002 \\
HD 136118 & 442.48 & 0.014 \\
HD 178911 B & 7.88 & 0.925 \\
HD 190228 & 2.57 & 0.777 \\
\hline
\end{tabular}

Notes.

${ }^{a}$ Residuals from one-planet fit.

b Residuals from two-planet fit.

FAP was computed using the analytic FAP formula of Horne \& Baliunas (1986). Of the 1000 trials, only 11 did not result in a successful recovery of the signal of the second planet. The correct period was recovered 995 times, and the FAP exceeded $0.1 \%$ only 6 times; the worst FAP was $0.3 \%$. These results indicate that our method should have been able to detect the signal of HD $74156 \mathrm{~d}$, had it been present with the parameters given by Bean et al. (2008). 

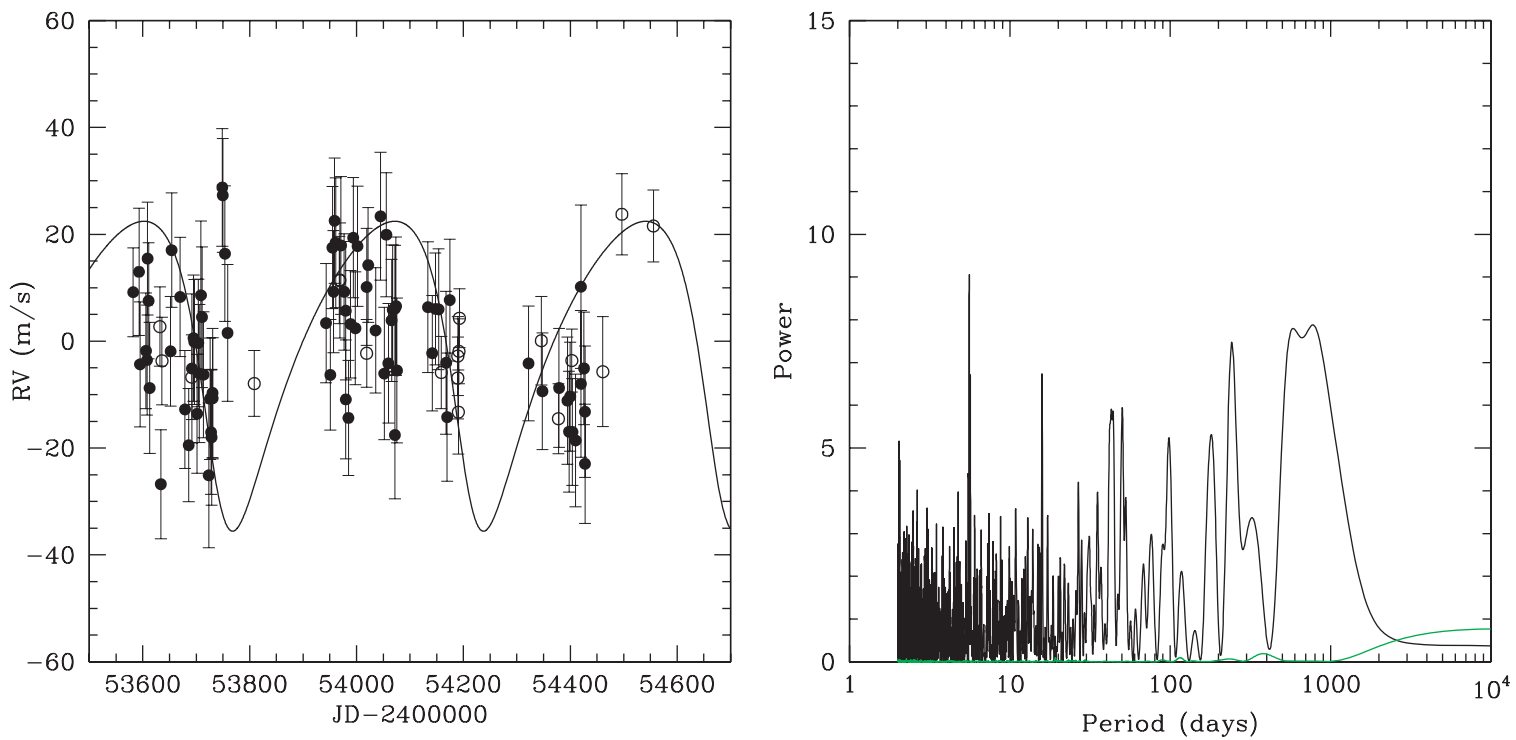

Figure 1. Left panel: radial-velocity data for HD 20367. Filled circles: HET, open circles: $2.7 \mathrm{~m}$. The Geneva group's orbital solution for the proposed planet is shown as a solid line. Right panel: Lomb-Scargle periodogram of the velocities. The 5.5-day stellar rotation period is evident, but no other periodicities are significant.

(A color version of this figure is available in the online journal.)

In Bean et al. (2008), the iodine-free stellar template spectrum was obtained at a resolving power of $R=120,000$, rather than the $R=60,000$ which is standard for targets in this paper. We obtained an $R=120,000$ template spectrum on 2007 November 12 , but the velocities computed using this template resulted in a two-planet fit with a slightly higher rms (HET: $8.9 \mathrm{~m} \mathrm{~s}^{-1}$ ) than the original $R=60,000$ template (HET: $8.3 \mathrm{~m} \mathrm{~s}^{-1}$ ). All analysis for HD 74156 in this paper refers to velocities obtained using the $R=60,000$ template.

A periodogram of the residuals to our two-planet fit is shown in the left panel of Figure 3, and those residuals are phased to the 346.6-day period in the right panel. The window function (gray dotted line) has a broad peak near 346 days due to the 1-year observing window. The phase gaps (right panel) are expected since the trial period is close to one year. No clear Keplerian signal is evident despite the large number of data points $(N=177)$. We conclude from these data that there is not sufficient evidence for a third planet in the HD 74156 system.

$47 U M a$ (= HD 95128). In Wittenmyer et al. (2007a), we performed these fits to an earlier set of data from McDonald Observatory. Those results did not provide convincing evidence for the outer planet reported by Fischer et al. (2002) at $P \sim 2594$ days; rather, we obtained a best-fit two-planet model with $P_{2} \sim 6900$ days. Here we include an additional 14 epochs from HET, and the best-fit two-planet model now calls for $P_{2} \sim 9660$ days. As in previous attempts to fit a second planet, the parameters $e_{2}$ and $\omega_{2}$ needed to be held fixed, at the values proposed by Fischer et al. (2002): $e_{2}=0.005$ and $\omega_{2}=127^{\circ}$. The rms about a single-planet model is $10.2 \mathrm{~m} \mathrm{~s}^{-1}$, compared to $8.6 \mathrm{~m} \mathrm{~s}^{-1}$ when a second planet is included. Considering the continued ambiguity in the parameters for a second planet, and the ever-lengthening period of such an object, we use the one-planet fit for all further analysis in this work.

HD 114783. Vogt et al. (2002) reported the planet orbiting HD 114783, and recently, Wright et al. (2007) proposed an outer companion with a period of at least 8 yr. Here, we combine the Keck data given in Butler et al. (2006) with HET observations. A single-planet fit has a total rms of $6.25 \mathrm{~m} \mathrm{~s}^{-1}$ and $\chi_{\nu}^{2}=4.91$, whereas a two-planet fit reduces the rms to $4.42 \mathrm{~m} \mathrm{~s}^{-1}$ and the $\chi_{v}^{2}$ to 1.81 . The data considered in Wright et al. (2007) were of

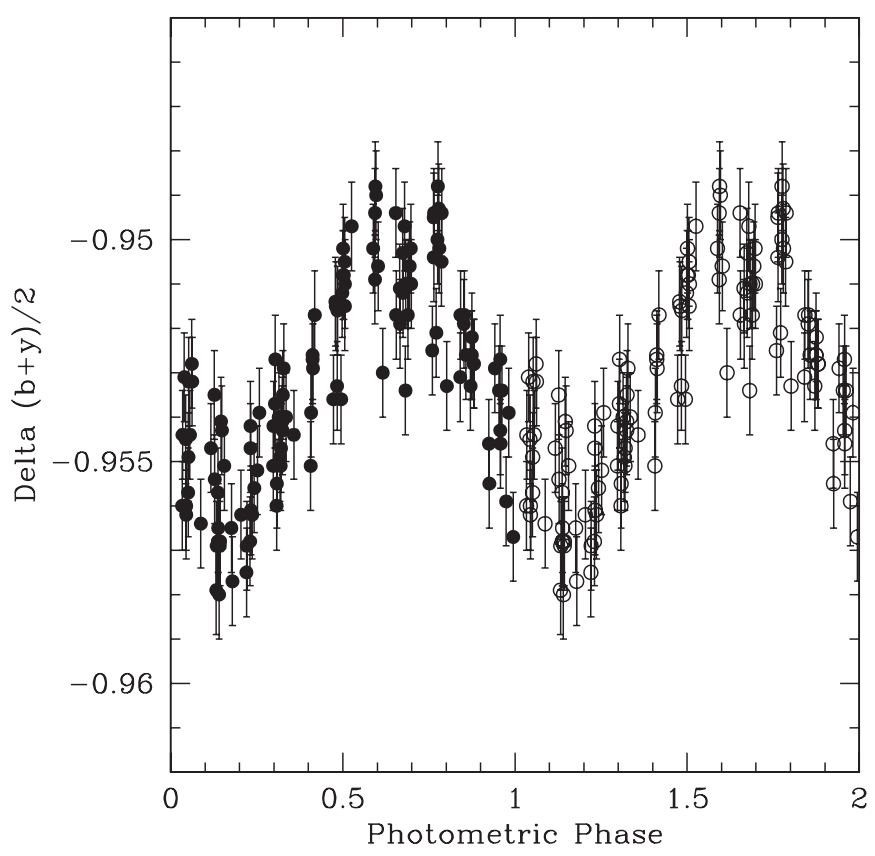

Figure 2. Photometric observations of HD 20367 phased to the stellar rotation period of 5.50 days. Two cycles are shown for clarity.

insufficient duration to establish a solution for the outer planet, but the combination of data allows for a Keplerian fit to converge. Although a 2-Keplerian model can be fitted to these data, it is of limited utility: the outer planet has a 50\% uncertainty in period $\left(P_{2}=5098 \pm 2576\right.$ days $)$. Our results support those of Wright et al. (2007), that a second object is likely present, although there is not yet a sufficient time baseline of observations to establish its nature. The one-planet fit was used to derive the parameters given in Table 3, and was also used for the detectionlimit determination in Section 5.

$H D$ 128311. The inner planet $(P \sim 450$ days $)$ in the HD 128311 system was first discovered by Butler et al. (2003), who noted a linear trend in the residuals to the fit, as well as 

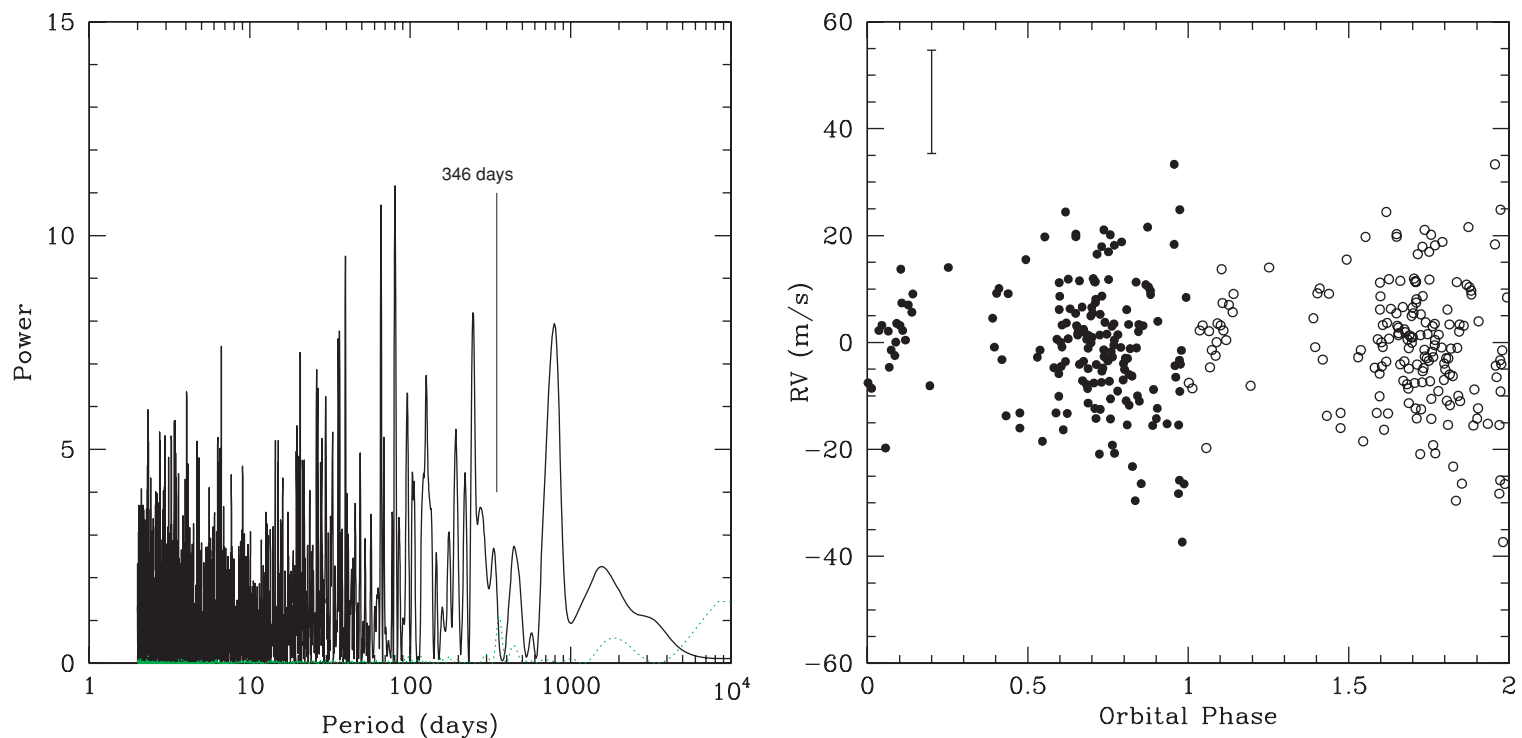

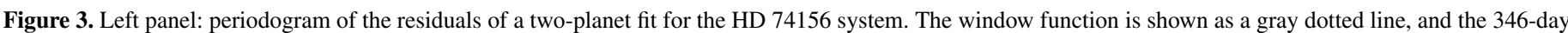

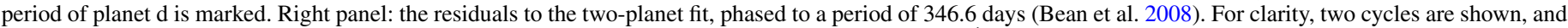
the error bars have been omitted. A reference error bar representing the mean uncertainty of $9.65 \mathrm{~m} \mathrm{~s}^{-1}$ is shown.

(A color version of this figure is available in the online journal.)
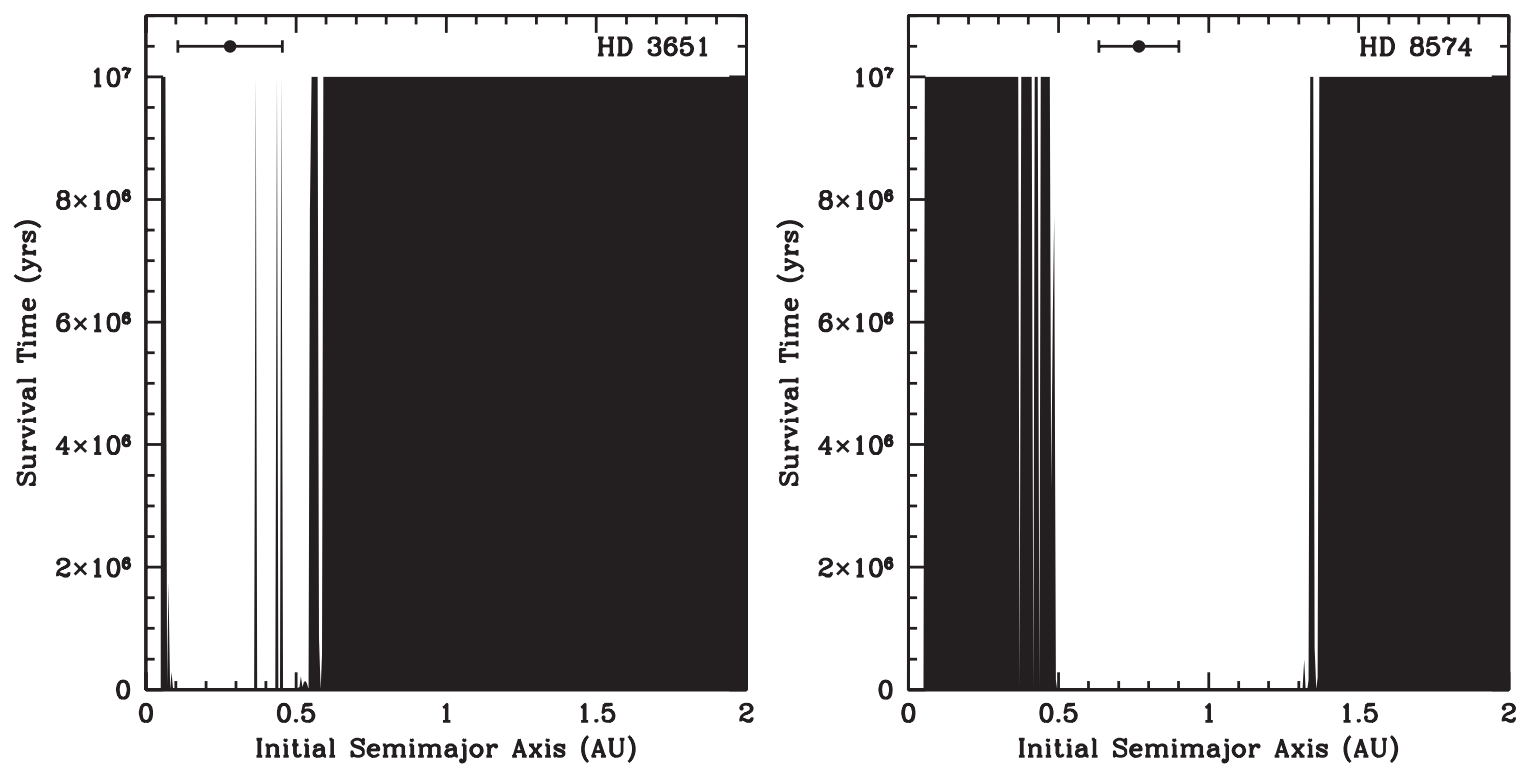

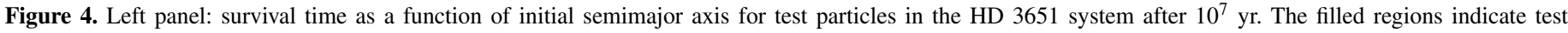

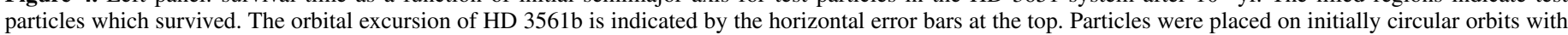
$0.05<a<2.00$ AU. Right panel: same, but for the HD 8574 system.

the extremely high activity level. Those authors estimated the stellar jitter at $30 \mathrm{~m} \mathrm{~s}^{-1}$, and expressed concern that the planetary signal may have its origin in the stellar velocity jitter. Additional data proved that the inner planet was indeed real, and Vogt et al. (2005) reported a second planet at the 2:1 mean-motion resonance (MMR). They published a solution consisting of two superposed Keplerian orbits, noting that preliminary dynamical tests showed the system to be unstable, and that the system was likely in a protected 2:1 resonance. Goździewski \& Konacki (2006), in their dynamical analysis of available radial-velocity data, suggested that the observed signal could be attributed to a 1:1 resonance, i.e., a pair of Trojan planets. In this work, we fit a double Keplerian model to the combined Keck and
HET data. Convergence is achieved, with a total rms of 16.9 $\mathrm{m} \mathrm{s}^{-1}$ about both data sets (Keck: $15.8 \mathrm{~m} \mathrm{~s}^{-1}$, HET: $17.9 \mathrm{~m}$ $\mathrm{s}^{-1}$ ). The residuals show a strong periodicity near 11.5 days, with bootstrap FAP less than $0.01 \%$. Photometry of HD 128311 by G. Henry in Vogt et al. (2005) indicates a stellar rotation period of 11.53 days with a photometric amplitude of 0.03 mag. Hence, it is quite clear that the residual signal is caused by stellar rotation in this highly active star.

HD 130322. HD 130322 is host to a hot Jupiter in a 10.7day period, discovered with the CORALIE observations of Udry et al. (2000). Four data sets are available for this object: CORALIE (Udry et al. 2000), Keck (Butler et al. 2006), HET, and $2.7 \mathrm{~m}$. Fitting all four sets together results in a total rms of 

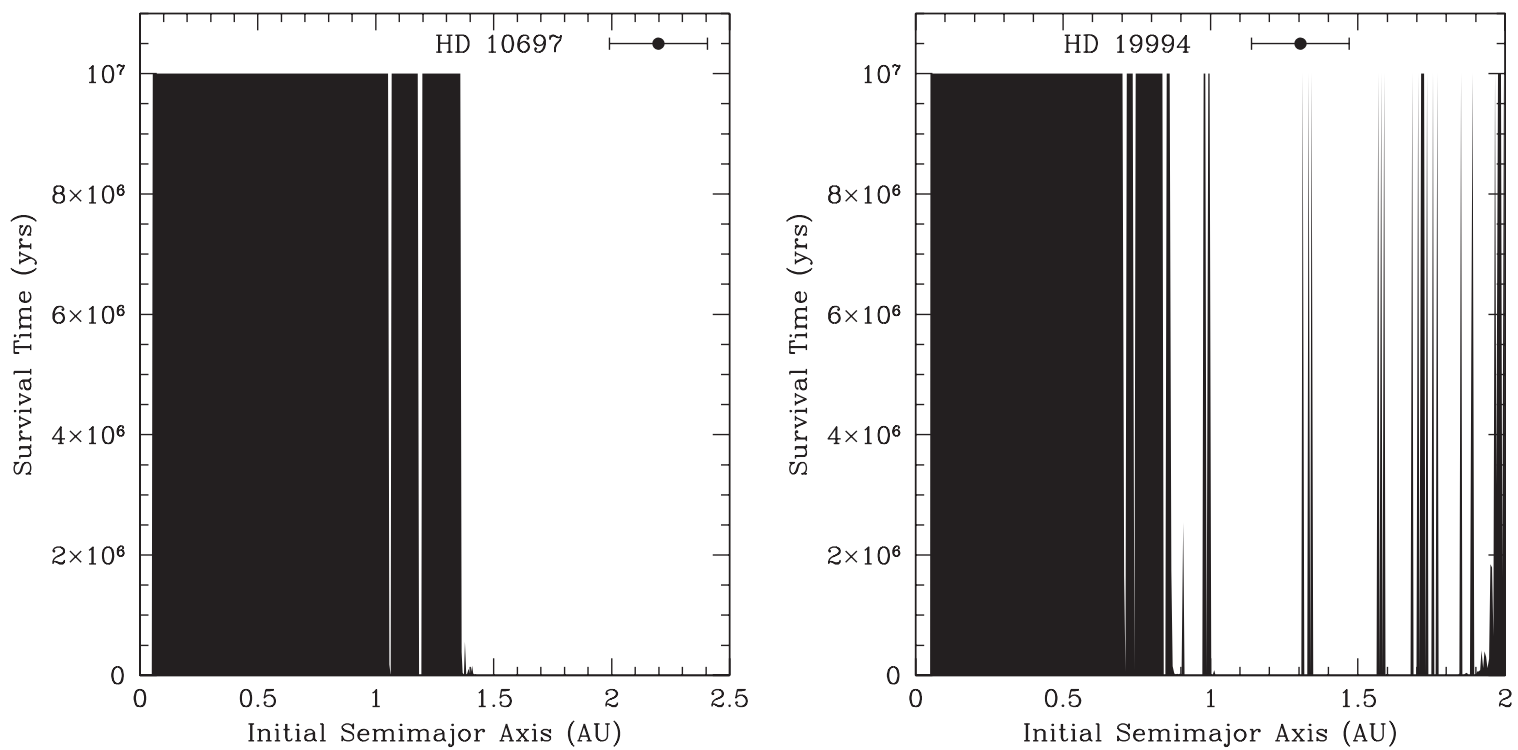

Figure 5. Same as Figure 4, but for the HD 10697 (left) and HD 19994 (right) systems.
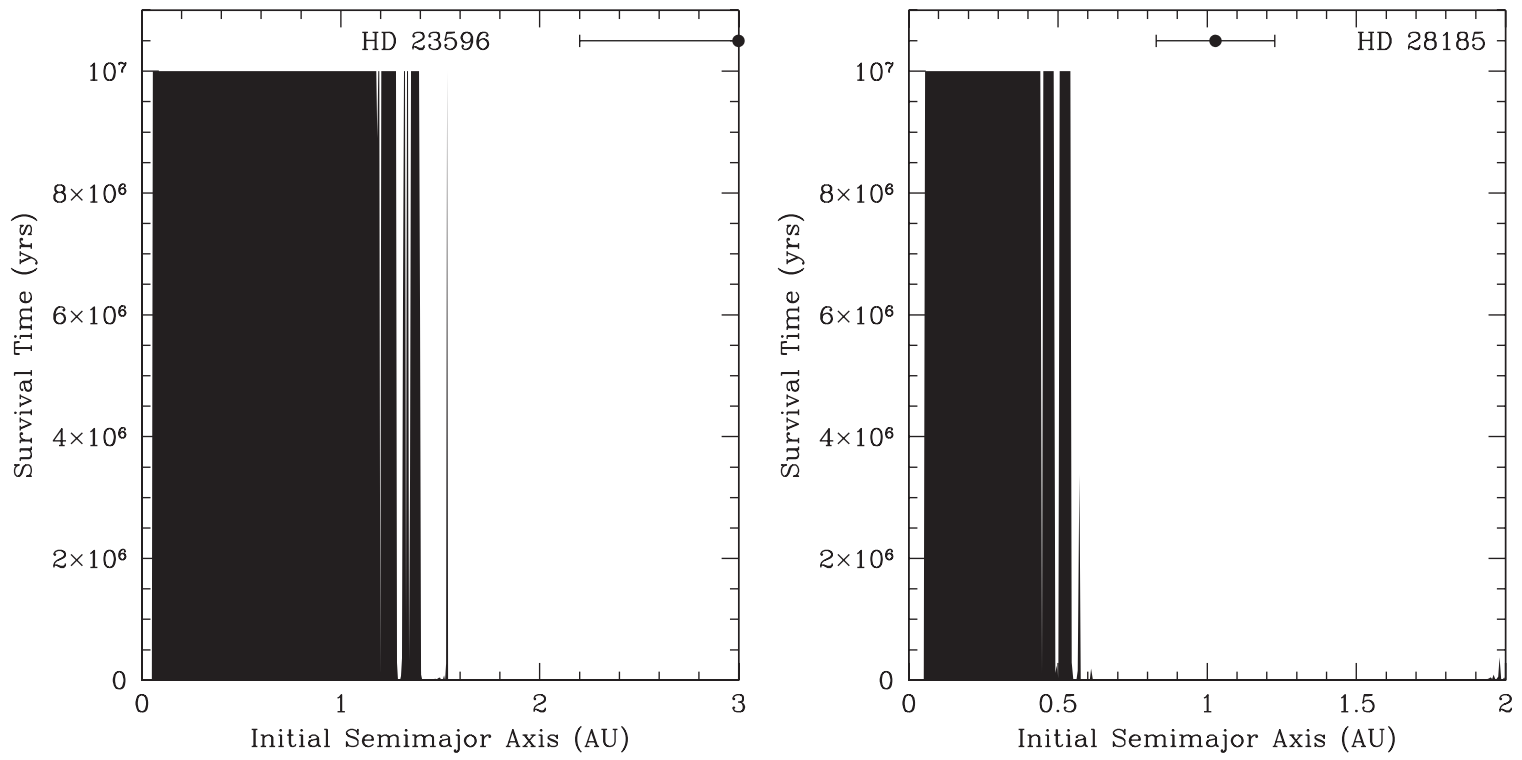

Figure 6. Same as Figure 4, but for the HD 23596 (left) and HD 28185 (right) systems.

$14.8 \mathrm{~m} \mathrm{~s}^{-1}$, but removing the CORALIE data drops the rms to $9.3 \mathrm{~m} \mathrm{~s}^{-1}$. In addition to the large scatter about the fit, a highly significant periodicity remains at 35 days (FAP $<0.01 \%$ ), which vanishes when the CORALIE data are removed. Due to these irregularities, we elect to exclude those data from the fits. The precision of the derived orbital parameters is not significantly affected by this removal, since the CORALIE data span only 167 days. For all further analysis in this work, we refer to the fit which excluded the CORALIE data. As given in Table 5, a residual period is present at $P \sim 438$ days (FAP $=0.16 \%$ ). However, the HET velocities obtained using a second iodinefree template spectrum show no such periodicity. Those results show a residual period at 2.518 days, with a bootstrap FAP of $0.35 \%$. A second planet can be fitted at this shorter period, and preliminary dynamical tests show that it would remain stable for at least $10^{7} \mathrm{yr}$; however, the disagreement between the two templates makes it imprudent for us to claim a detection at this time.

\section{DYNAMICAL MAPPING}

With the increasing availability of computing power and planetary systems, many investigators have undertaken $N$ body simulations of known planetary systems in an effort to characterize regions in which additional bodies could be found. Menou \& Tabachnik (2003) performed a comprehensive testparticle analysis of 85 systems to determine the extent of the habitable zones in the presence of the known planet(s). Due to disruptions from the known giant planet's "zone of influence," they found that only one-fourth of the systems had dynamical habitability comparable to our own solar system. In addition to test particles, massive "test planets" have also been used to test observational claims for new planets and to probe known multiple-planet systems for additional regions of stability (Rivera \& Lissauer 2000, 2001; Raymond \& Barnes 2005; Rivera \& Haghighipour 2007). Likewise, in this section we perform test-particle and massive-body simulations on the 

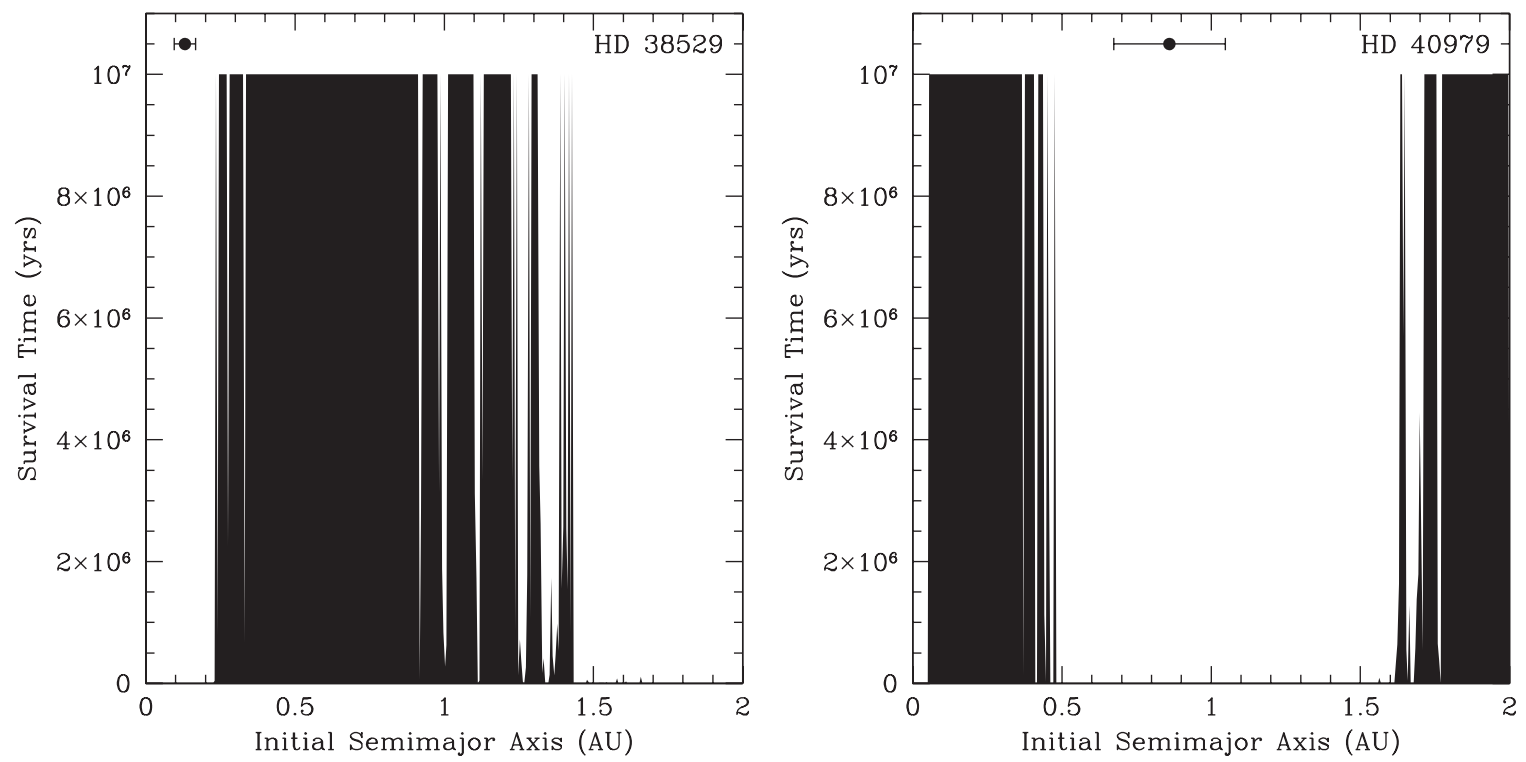

Figure 7. Same as Figure 4, but for the HD 38529 (left) and HD 40979 (right) systems.
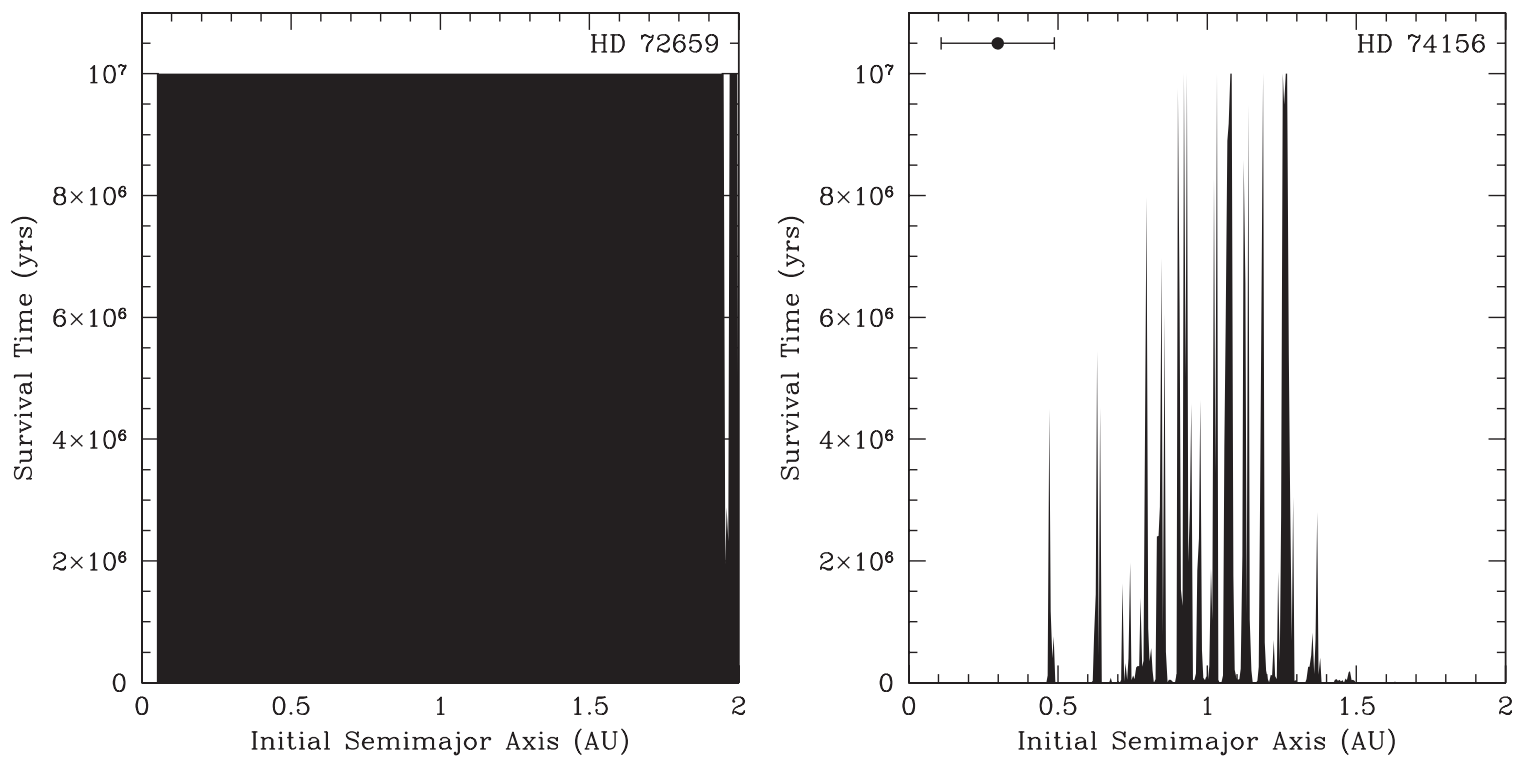

Figure 8. Same as Figure 4, but for the HD 72659 (left) and HD 74156 (right) systems. HD 72659b, with an orbital excursion of 3.48-6.48 AU, is off the plot. The recently announced planet HD $74156 \mathrm{~d}$, between planets b and c, was not included in the simulation, but would reside in the narrow stable strip.

systems targeted by the intensive radial-velocity monitoring described in Section 3.

\subsection{Test-Particle Simulations}

We performed test-particle simulations using SWIFT $^{6}$ (Levison \& Duncan 1994) to investigate the dynamical possibility of additional low-mass planets in each of the systems considered here. SWIFT is a numerical integration package which is designed to solve the equations of motion for gravitational interactions between massive bodies (star, planets) and massless test particles. Neptune-mass planets can be treated as test particles ( 1 Neptune mass $=0.054 M_{\text {Jup }}$ ) since the exchange of angular momentum with jovian planets is small. We chose the regularized mixed-variable symplectic integrator (RMVS3) version

SWIFT is publicly available at http://www.boulder.swri.edu/ hal/swift.html of SWIFT for its ability to handle close approaches between massless, noninteracting test particles, and planets. This version is most efficient when the gravitational interactions are dominated by a single body (the central star). A symplectic integrator has the advantage that errors in energy and angular momentum do not accumulate. Particles are removed if they are (1) closer than 1 Hill radius to the planet, (2) closer than $0.01 \mathrm{AU}$ to the star, or (3) farther than $10 \mathrm{AU}$ from the star. A planetary-mass object passing within 1 Hill radius of another planet, or within $0.01 \mathrm{AU}\left(2 R_{\odot}\right)$ of the star's barycenter, is unlikely to survive the encounter. Since the purpose of these simulations is to determine the regions in which additional planets could remain in stable orbits, we set the outer boundary at 10 AU because the current repository of radial-velocity data cannot detect objects at such distances.

For each planetary system, 390 test particles were placed in initially circular orbits, spaced every $0.005 \mathrm{AU}$ in the region between 0.05 and 2.0 AU. We have chosen to focus on this 

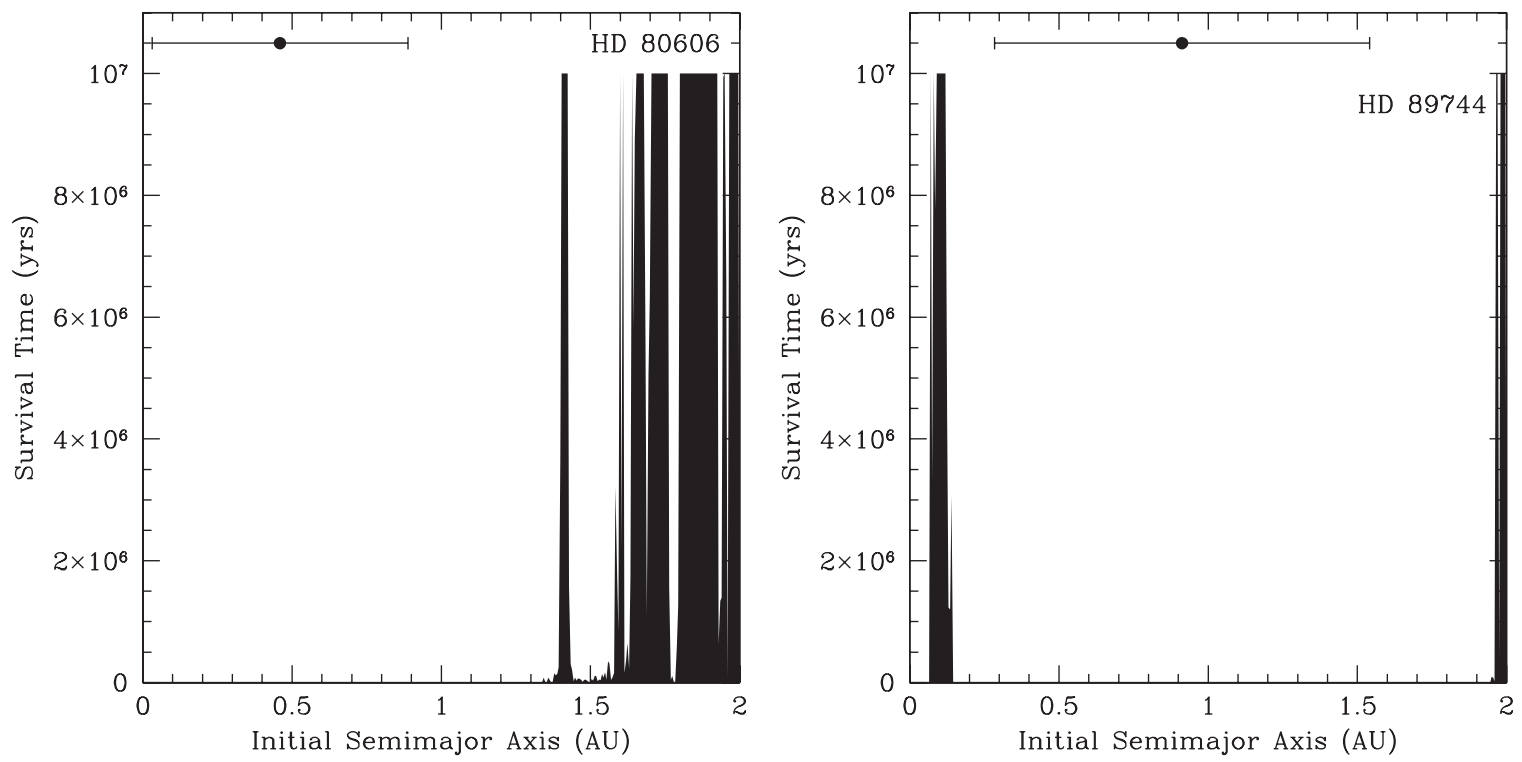

Figure 9. Same as Figure 4, but for the HD 80606 (left) and HD 89744 (right) systems.
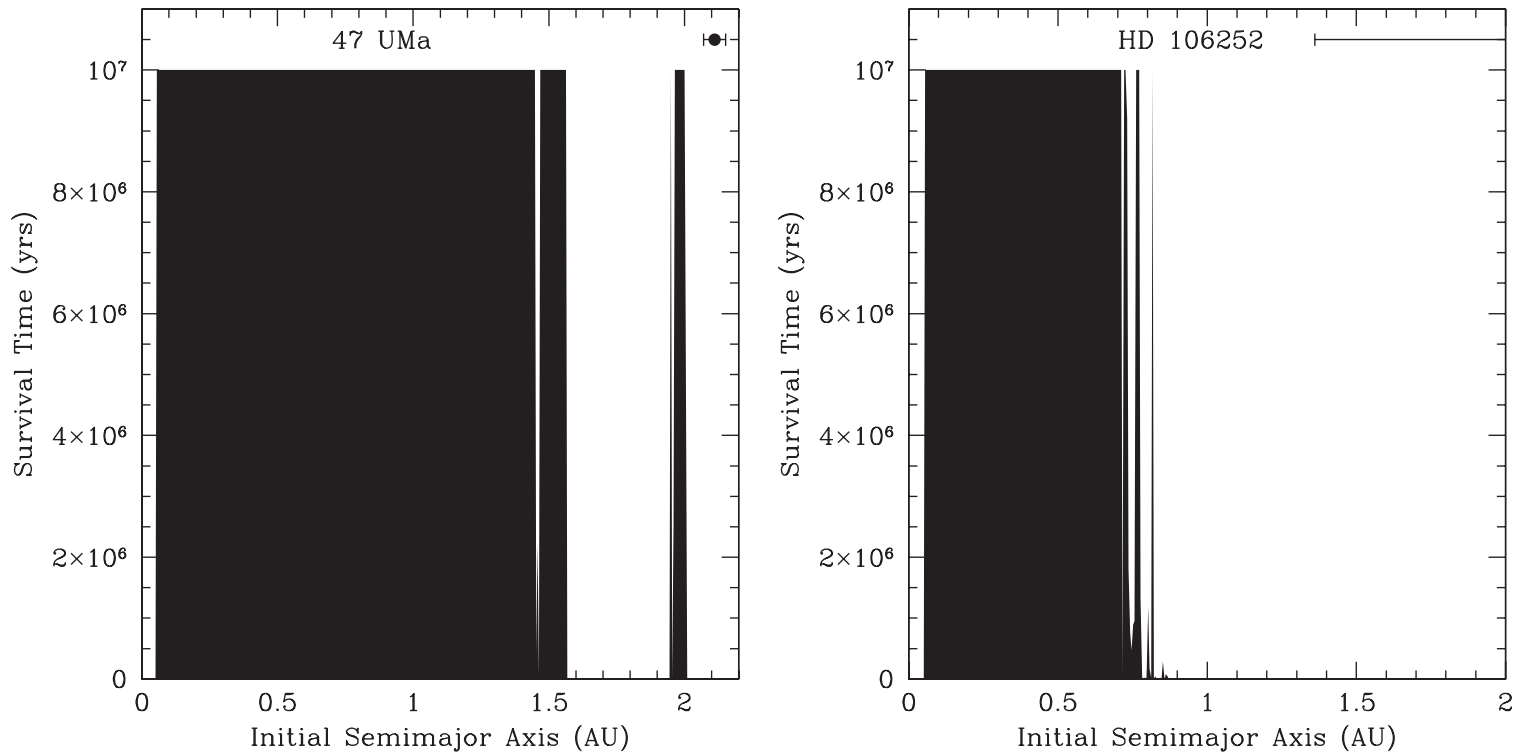

Figure 10. Same as Figure 4, but for the $47 \mathrm{UMa}$ (left) and HD 106252 (right) systems. Only $47 \mathrm{UMa}$ b was considered in the simulations. An outer body would be too distant to affect the region under consideration.

region because the duration of our high-precision HET data is currently 3-four years for the objects in this study. The test particles were coplanar with the existing planet, which had the effect of confining the simulation to two dimensions. The initial orbital positions of the particles were randomly distributed in orbital phase with respect to the existing planets. The method used here are the same as Wittenmyer et al. (2007b), in which we performed test-particle simulations for six highly eccentric planetary systems. Input physical parameters (Table 3) for the known planet in each system were obtained from our Keplerian orbit fits combining published velocity data and new observations from McDonald Observatory. The planetary masses were taken to be their minimum values $(\sin i=1)$. By choosing the minimum mass for the planets, the regions of dynamical stability shown by the test-particle results are larger. Since the system inclinations are almost certainly not edgeon, and hence the true planetary masses are higher, we expect the actual regions of stability to be smaller than shown here.
The systems were integrated for $10^{7} \mathrm{yr}$, following Barnes \& Raymond (2004) and allowing completion of the computations in a reasonable time. We observed that nearly all of the testparticle removals occurred within the first $10^{6} \mathrm{yr}$; after this time, the simulations had essentially stabilized to their final configurations.

\subsection{Test-Particle Results}

The results of the test-particle simulations are shown in Figures 4-13. The survival time of the test particles is plotted against their initial semimajor axis. Two systems targeted by the radial-velocity observations were not included in these simulations: HD 20367, because there is no evidence for a planet, and HD 128311, since the Keplerian orbit solution obtained in Section 3.1 results in an unstable system. As shown in Figure 4, the short-period planet HD $3651 \mathrm{~b}$ sweeps clean the region inside of about 0.5 AU. However, a small number of test 

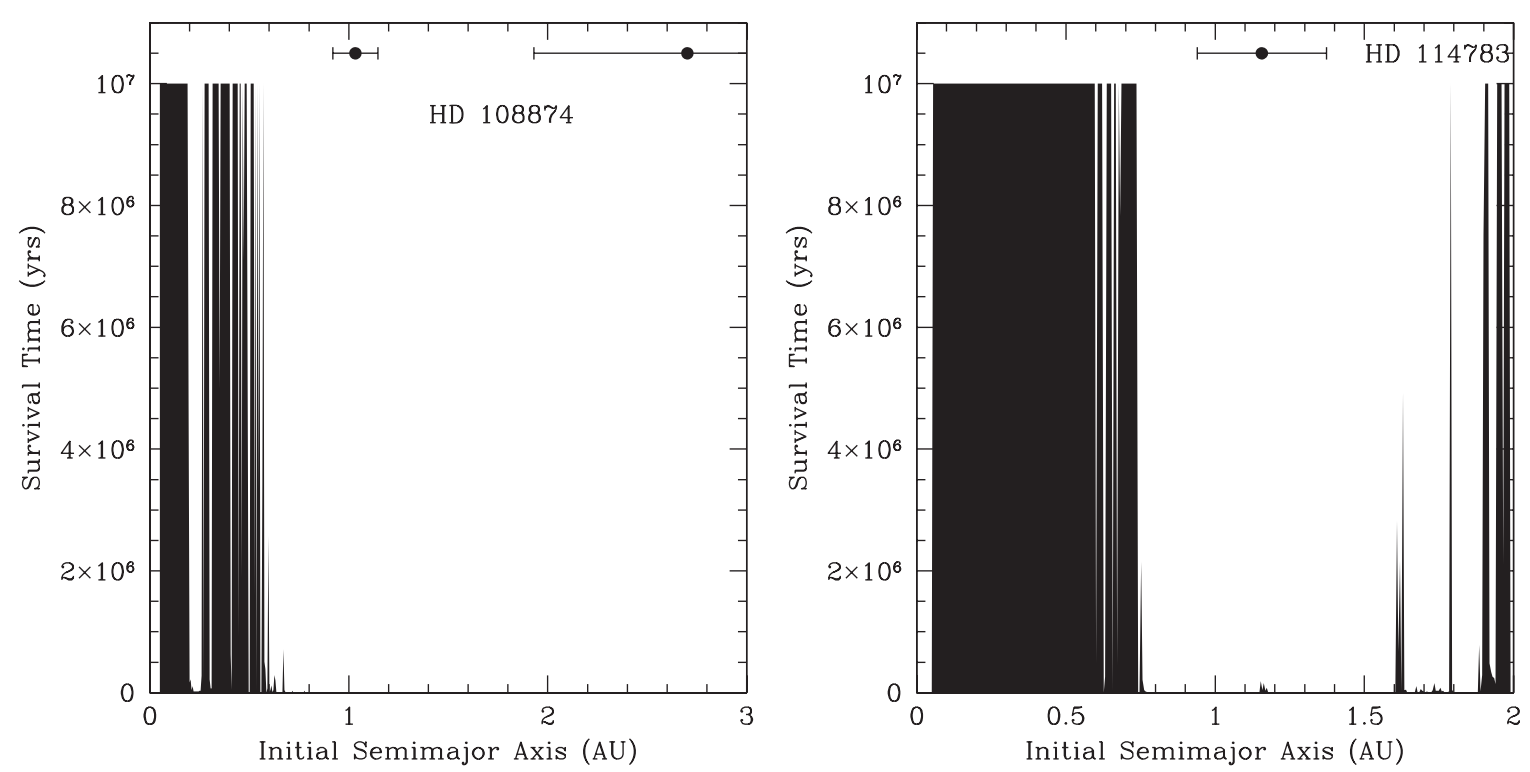

Figure 11. Same as Figure 4, but for the HD 108874 (left) and HD 114783 (right) systems.
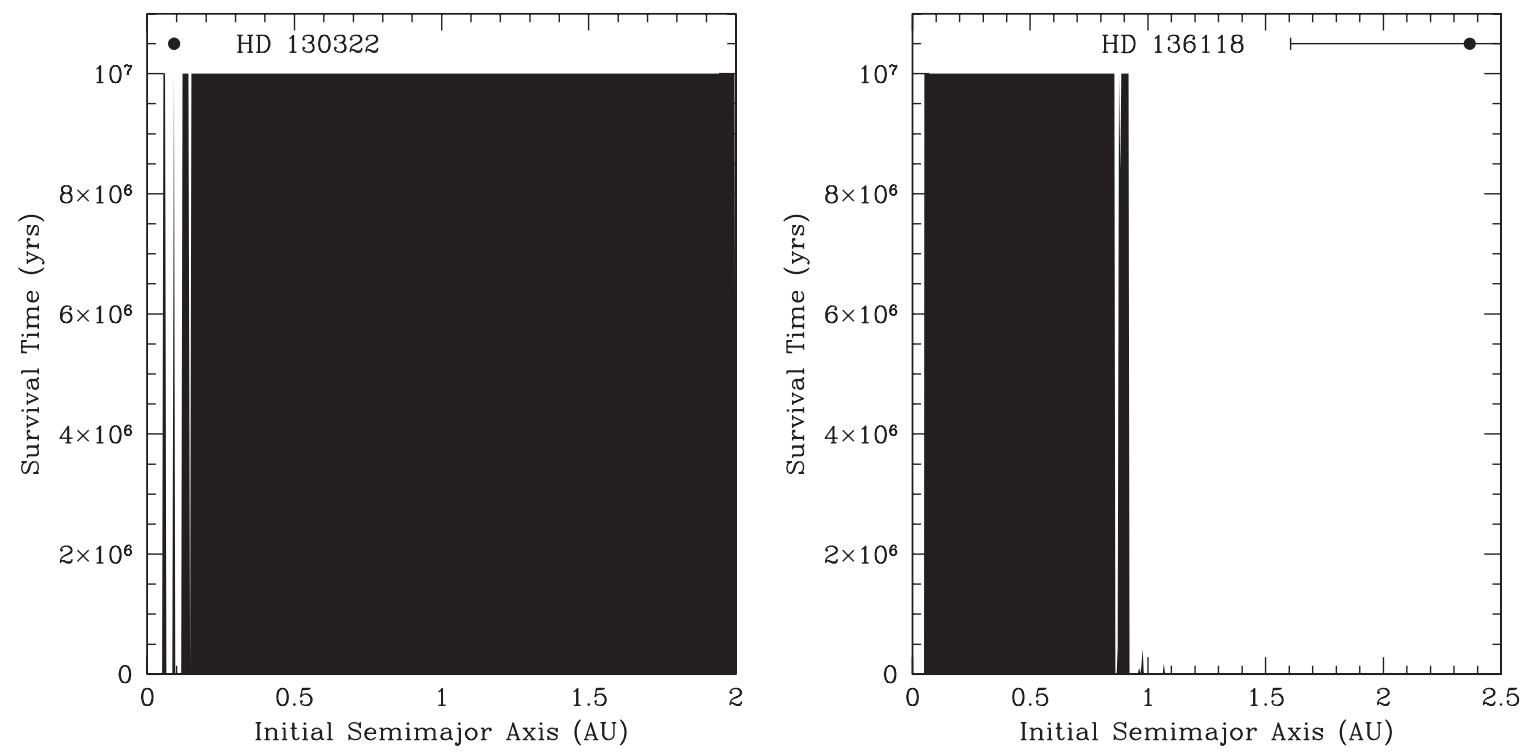

Figure 12. Same as Figure 4, but for the HD 130322 (left) and HD 136118 (right) systems.

particles remained in low-eccentricity orbits near the 1:3 and 2:1 MMRs. Since these regions lie within the orbital excursion of HD $3651 \mathrm{~b}$, these appear to be protected resonances. The eccentricity of the test particles in the region of the 1:3 MMR oscillated between 0.00 and 0.31 with a periodicity of about $1.2 \times 10^{5} \mathrm{yr}$, while those in the 2:1 resonance remained at $e \lesssim 0.07$ throughout the simulation. All particles beyond about $0.6 \mathrm{AU}$ also remained in stable orbits, which is not surprising given the low mass of the planet. In simulations by Mandell et al. (2007) and Raymond et al. (2006), a migrating Jupitermass planet captured planetesimals into low-order resonances, and these accreted into terrestrial planets during the $200 \mathrm{Myr}$ run. The architecture of the HD 3651 system, with a $0.2 M_{\text {Jup }}$ planet at $0.3 \mathrm{AU}$, is similar to the configuration modeled by Mandell et al. (2007). Given the stable regions evident near the 1:3 and 2:1 resonances for HD $3651 \mathrm{~b}$, it is possible that terrestrial-mass planets were captured into these regions during the migration process. The detection limits for HD 3651 (Section 5) complement the dynamics well, and the current data can place upper limits of 1-2 Neptune masses (17-34 Earth masses) on such objects.

For most of the systems, the test-particle results give few surprises. Broad stable regions exist interior and exterior to HD $8574 \mathrm{~b}$, with the inner $0.47 \mathrm{AU}$ retaining $100 \%$ of particles. For HD 10697 and HD 23596, particles remained in the inner 1.35 AU and 1.4 AU, respectively. The HD 19994 system, as shown in Figure 5, proved to be quite interesting. One would expect any particles in orbits which cross that of the planet to be removed straightaway, but a few particles remained near the 1:1 resonance with the planet, in the range 1.29-1.33 AU. Laughlin \& Chambers (2002) investigated the possibility of planets in a 1:1 resonance, and concluded that such configurations are indeed possible. In the "eccentric resonance," one 1:1 configuration described by Laughlin \& Chambers (2002), one planet is in a nearly circular orbit while the other is in a highly eccentric orbit. Though the orbits cross, the longitudes of pericenter are sufficiently different to avoid close encounters. 

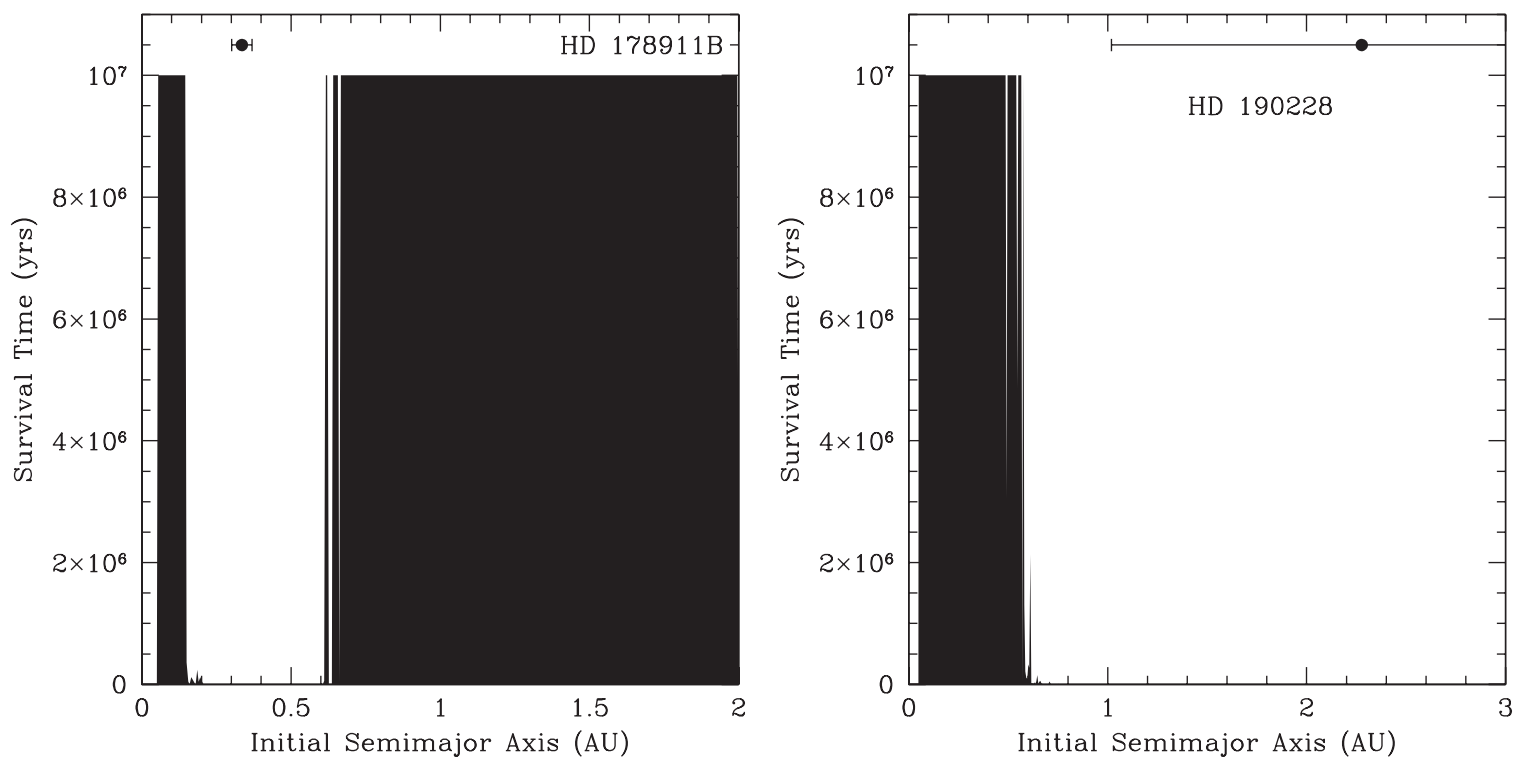

Figure 13. Same as Figure 4, but for the HD 178911 B (left) and HD 190228 (right) systems.

In the HD 28185 system (Figure 6), no stable regions exist exterior to the planet out to the maximum separation tested $(a=2.0 \mathrm{AU})$. Figure 7 shows the results for the HD 38529 and HD 40979 systems. There is a broad region of stability between the widely separated planets HD 38529b and c, consistent with the results of Barnes \& Raymond (2004). The outer planet does not fall within the range of Figure 7, but has an orbital excursion of 2.43-4.99 AU. For HD 74156, the recently announced planet $\mathrm{d}$ (Bean et al. 2008) in a 346-day period between planets $b$ and $\mathrm{c}$ was not included in the simulation. Only those particles in a narrow strip near 1.25 AU survived the full $10 \mathrm{Myr}$; planet d would fall within the stable region.

The 47 UMa system (Figure 10) included only the inner planet ( $a=2.11 \mathrm{AU}$ ) for this experiment. The parameters of an outer planet are highly uncertain (Wittenmyer et al. 2007a; Naef et al. 2004), and such an object would be too distant to affect the inner 2 AU explored here. A large region interior to the planet is stable for the full duration, including the habitable zone. This result is consistent with that of Jones et al. (2001), who also found the $47 \mathrm{UMa}$ habitable zone to be stable for an Earth-mass planet at $1 \mathrm{AU}$. With an $M \sin i$ of $6.9 M_{\text {Jup }}$, HD $106252 \mathrm{~b}$ clears out all particles outside of $a \sim 0.7$ AU. For the HD 108874 system, no test particles survive between the two planets (Figure 11), but those in the innermost $0.3 \mathrm{AU}$ remain stable. Particles interior to HD $114783 \mathrm{~b}$ were stable to about $a \sim 0.7 \mathrm{AU}$. As expected for the HD 130322 hot-Jupiter system, all particles with $a>0.15$ AU survived (Figure 12). In the HD 178911 B system (Figure 13), some particles remained in the inner 0.1 AU despite the large mass $\left(M \sin i=6.95 M_{\mathrm{Jup}}\right)$ and relative proximity $(a=0.34 \mathrm{AU})$ of the planet.

\subsection{Massive-Body Simulations}

Regions stable for massless test particles may not be stable for massive bodies. Alternatively, regions unstable for test particles may be able to host a massive planet. In the latter case, the existing planet(s) may adjust their orbits in response to the perturbation induced by the introduced planet. For these reasons, it is also important to consider the effect of massive "test planets" in order to obtain a more complete dynamical picture of the systems under consideration. In this section, we explore

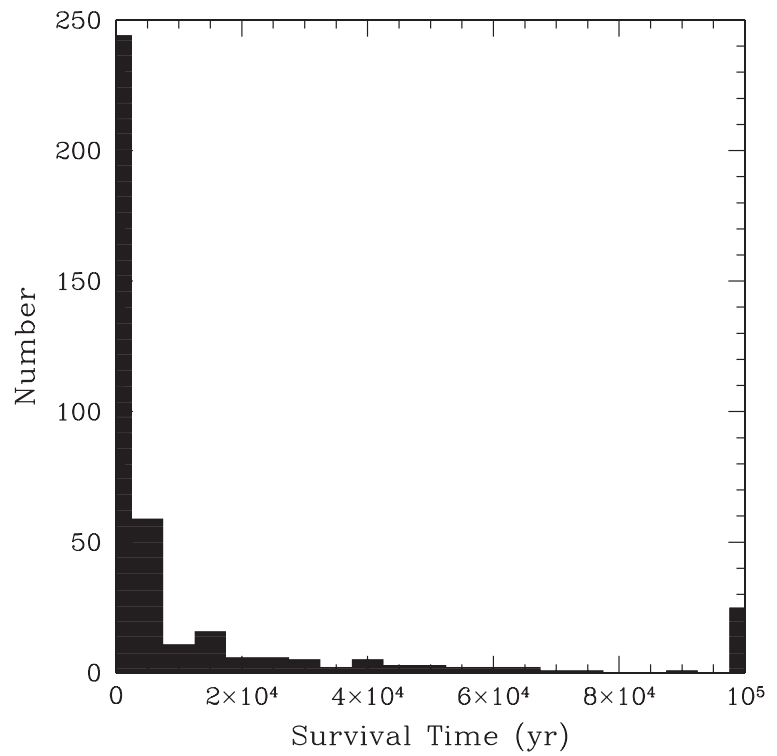

Figure 14. Histogram of the survival times for the unstable test configurations $(N=352)$. 20 realizations survived longer than $10^{5} \mathrm{yr}$.

the effect of inserting massive bodies into a known planetary system.

SWIFT's RMVS3 integrator cannot handle close encounters, when massive bodies are closer to each other than 3 Hill radii. For the massive-body simulations, we use the Mercury orbital integrator (Chambers 1999), which has a hybrid feature that switches from a mixed-variable symplectic integration to a Bulirsch-Stoer method when objects are within 3 Hill radii of each other. General relativistic effects have not been included. For these tests, fictitious planets were placed in each system on initially circular orbits at 0.05 AU intervals from 0.05 to 2.00 AU. The masses of the bodies were set at a Saturn mass (=0.3 $M_{\text {Jup }}$ ); this is comparable to the mass detectable by the radial-velocity survey, and is the mass used by Raymond \& Barnes (2005) in a similar investigation. These simulations ran for $10^{6} \mathrm{yr}$, and we observed that unstable configurations usually resulted in system destruction within $10^{5} \mathrm{yr}$. Figure 14 shows a histogram of the survival times for the unstable trials. 

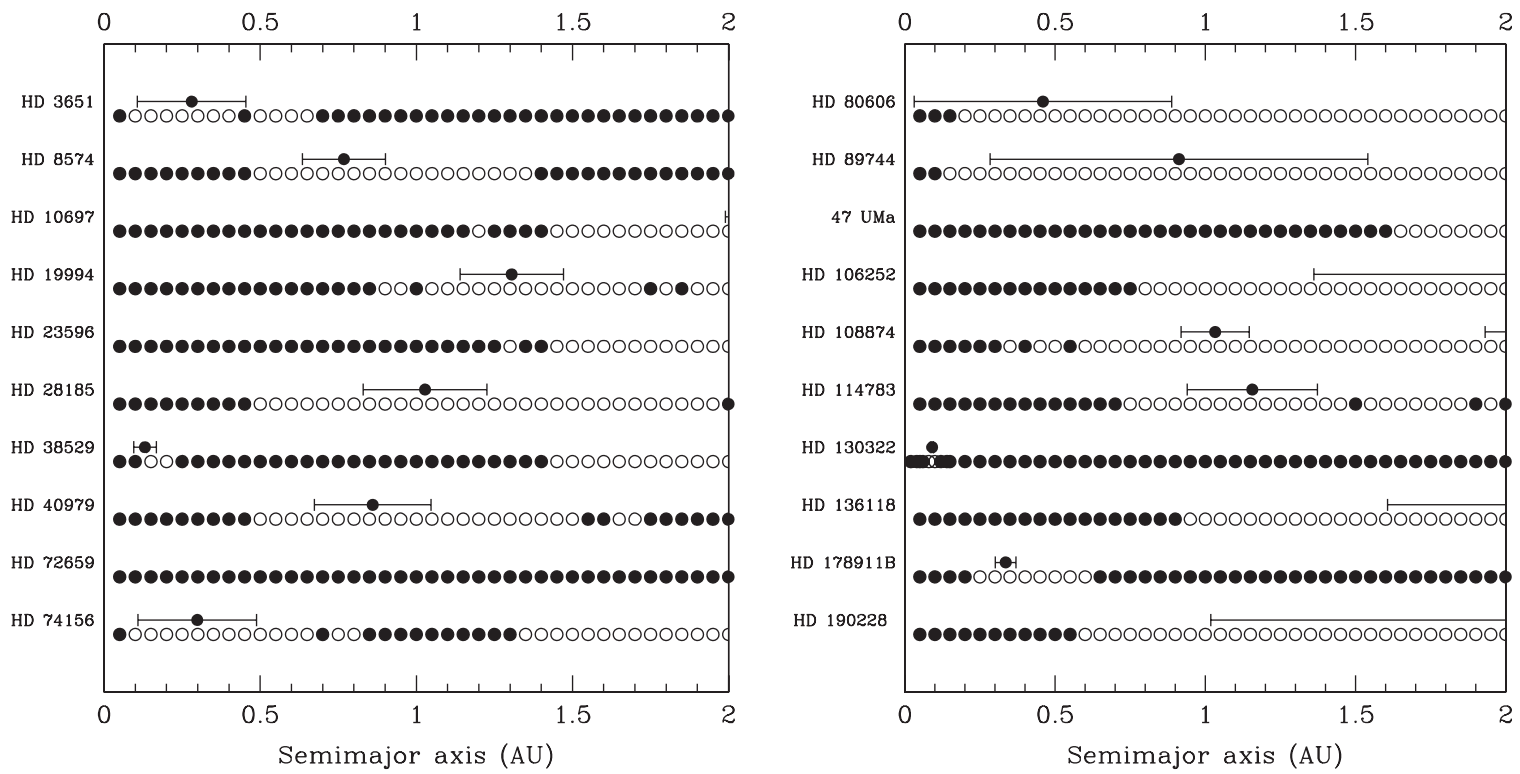

Figure 15. Survival of Saturn-mass planets for $10^{6} \mathrm{yr}$ on initially circular orbits in 20 planetary systems. The orbital excursions of the existing planets are indicated by the horizontal error bars. The open circles represent unstable locations, and the filled circles were stable for $10^{6} \mathrm{yr}$.
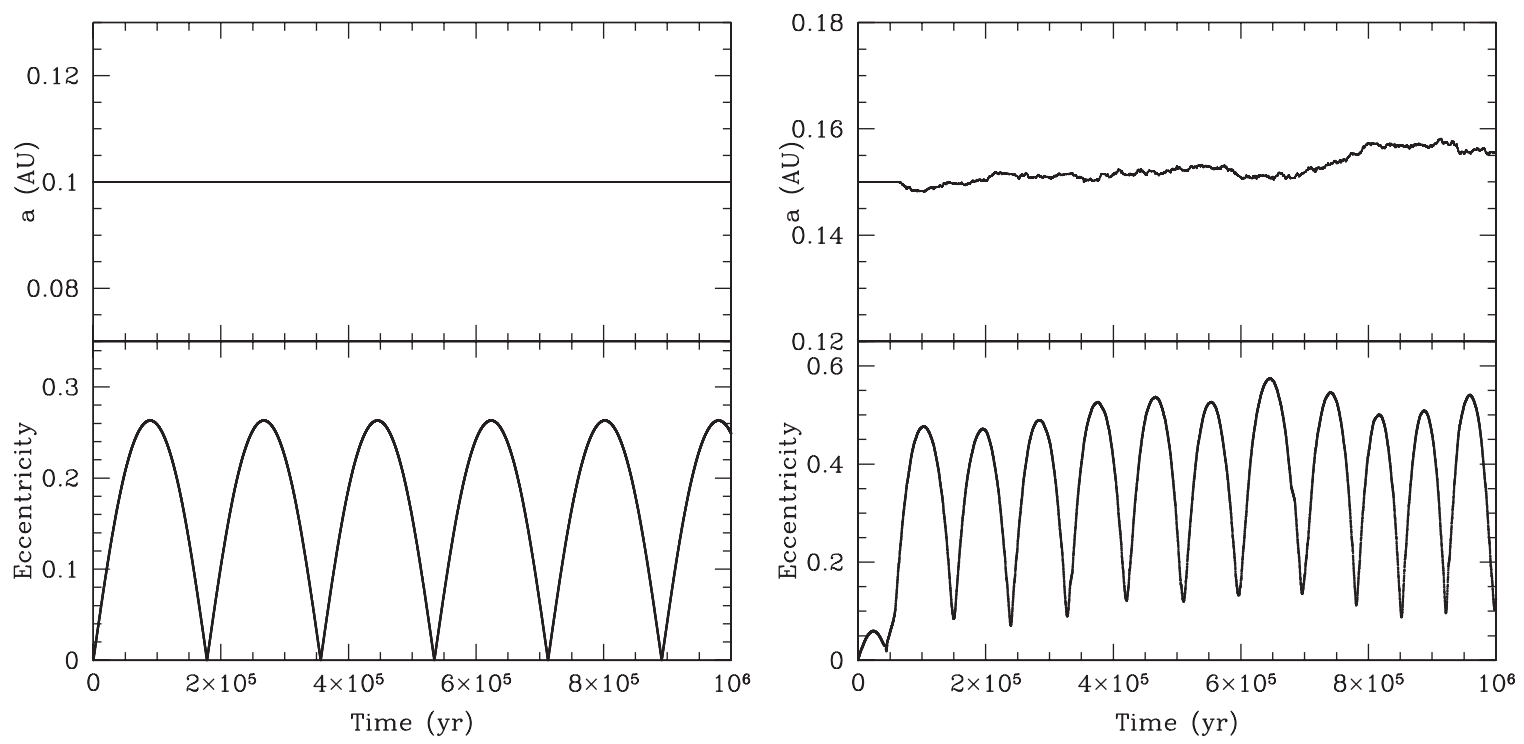

Figure 16. Left panel: behavior of the semimajor axis (top) and eccentricity (bottom) of a Saturn-mass test planet starting at $a=0.10$ AU in the HD 80606 system over a $10^{6} \mathrm{yr}$ period. Right panel: same, but for an object starting at $a=0.15 \mathrm{AU}$, which was then ejected at $t=5.7 \times 10^{6} \mathrm{yr}$.

\subsection{Massive-Body Results}

The results of the massive-body simulations are shown in Figure 15. The filled circles indicate test planets which remained throughout the $10^{6} \mathrm{yr}$ integration. For most of the systems, the regions stable for test particles are also stable for Saturnmass planets. For HD 3651 and HD 80606, some test planets which crossed orbits with the known planet survived. The 2:1 resonance of HD $3651 \mathrm{~b}(a \sim 0.45 \mathrm{AU})$ retained the Saturn-mass planet for $10^{6} \mathrm{yr}$, although its eccentricity varied chaotically, reaching $e \sim 0.22$. The HD 80606 system gave the most unexpected result: Saturn-mass planets remained in the region $a \leqslant 0.15 \mathrm{AU}$, despite crossing orbits with HD $80606 \mathrm{~b}$. The test planets at $0.05,0.10$, and $0.15 \mathrm{AU}$ reached maximum eccentricities of $0.13,0.26$, and 0.57 , respectively. For the test planets at 0.05 and $0.10 \mathrm{AU}$, the oscillations in eccentricity were regular in period and constant in amplitude, whereas for $a=0.15 \mathrm{AU}$, the oscillations varied in period and increased in amplitude toward the end of the $10^{6} \mathrm{yr}$ simulation (Figure 16). For the two cases in which the test planets exhibited irregular variations in eccentricity, the simulations were continued for $10^{7} \mathrm{yr}$, anticipating the eventual destruction of the system. The test planet at $0.45 \mathrm{AU}$ in the $\mathrm{HD} 3651$ system caused the ejection of HD $3651 \mathrm{~b}$ after $1.8 \times 10^{6} \mathrm{yr}$. Likewise, the test planet at $0.15 \mathrm{AU}$ in the HD 80606 system was ejected after $5.7 \times 10^{6} \mathrm{yr}$.

\section{DETECTION LIMITS}

\subsection{Methods}

In Wittenmyer et al. (2006), we described a detection-limit algorithm implemented on the sample of constant stars from the long-term planet search at McDonald Observatory. This approach was based on that used by Endl et al. (2002) to derive detection limits from their survey with the ESO Coude 

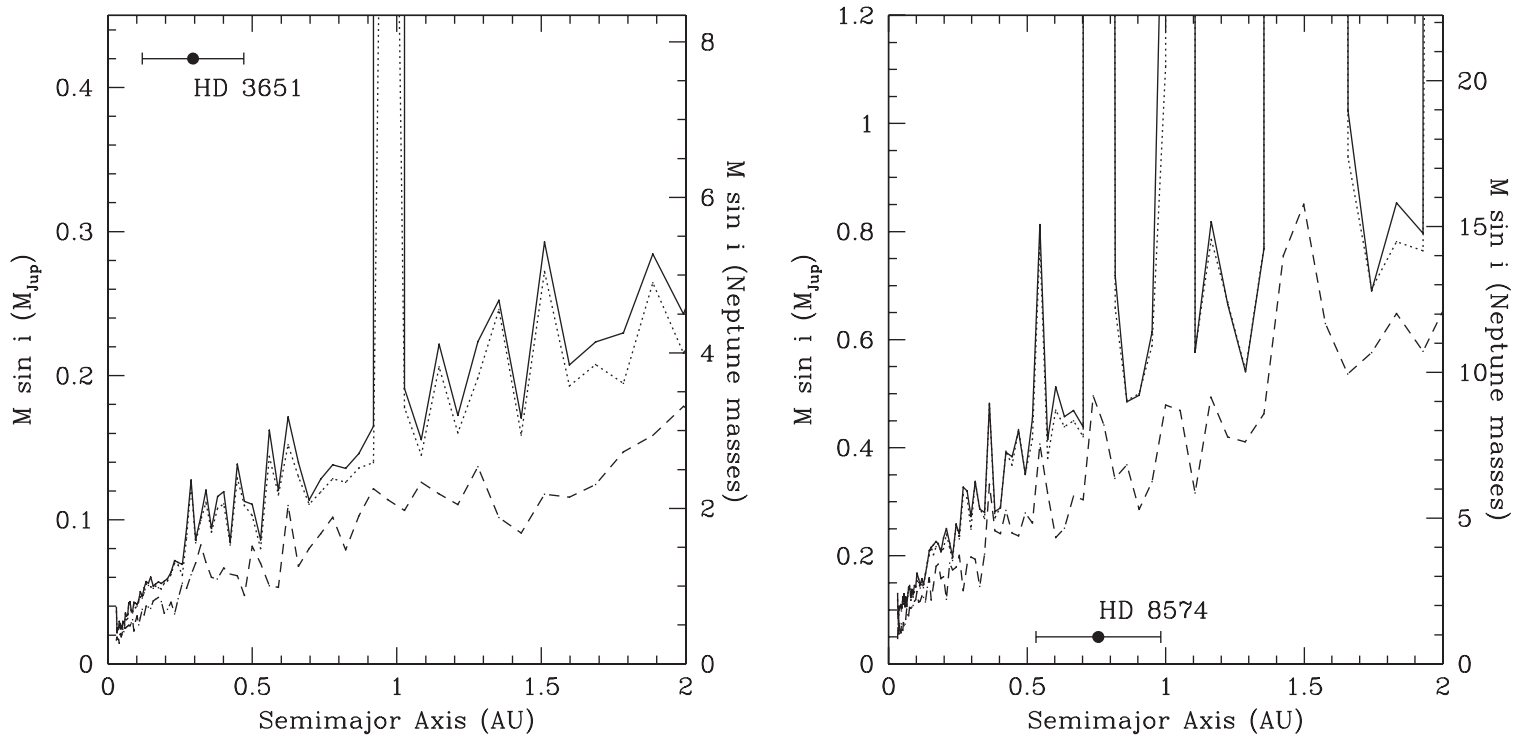

Figure 17. Left panel: detection limits for additional planets in orbits with $e=0.20$ in the HD 3651 system (solid line). This value represents the mean eccentricity of surviving test particles from the dynamical simulations discussed in Section 4. Planets in the parameter space above the solid line are excluded at the 99\% confidence level. Limits for planets in circular orbits are shown as dotted (99\% recovery) and dashed (50\% recovery) lines. Right panel: same, but for HD 8574 (solid line: $e=0.10)$.
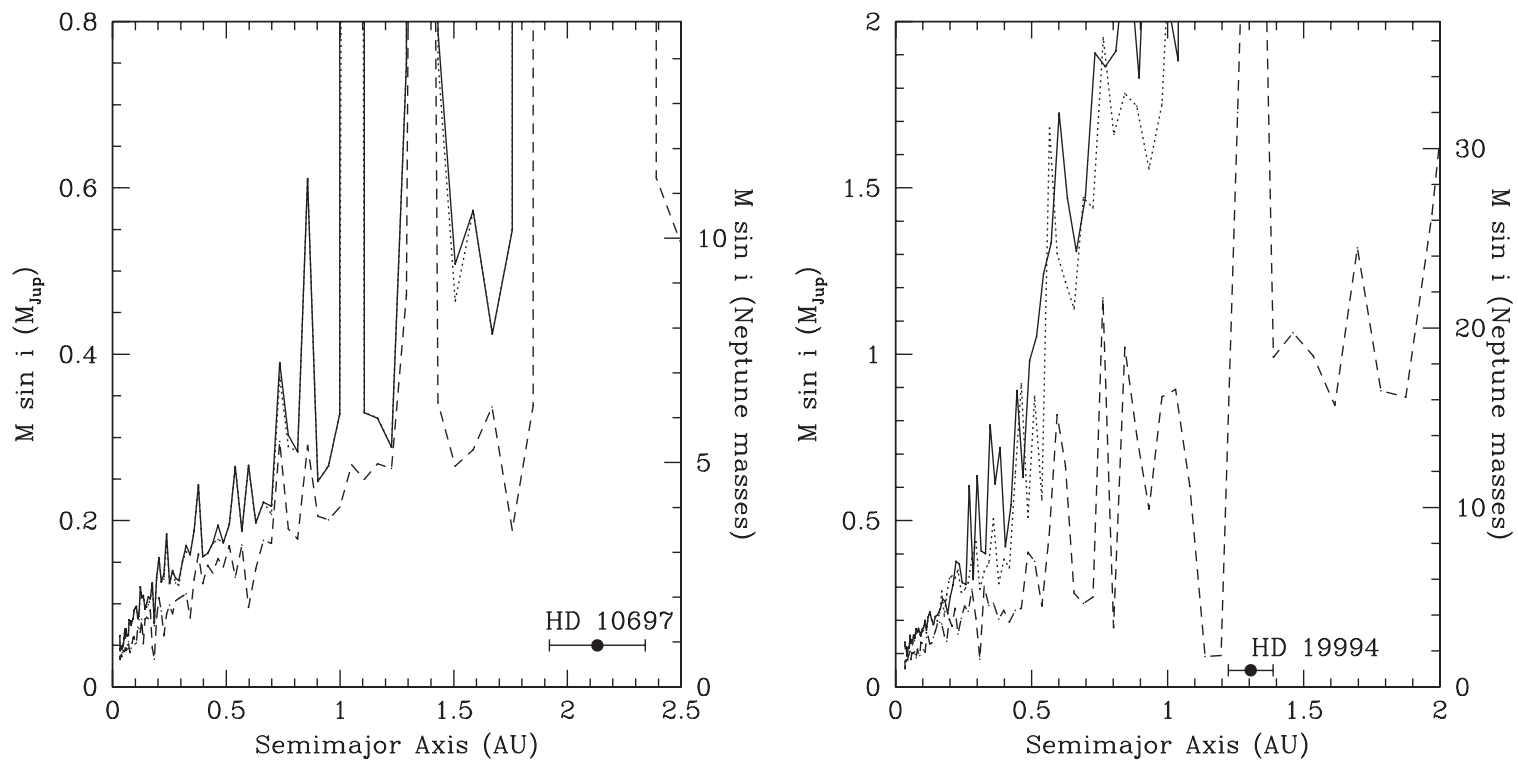

Figure 18. Left panel: same as Figure 17, but for HD 10697 (solid line: $e=0.04$ ). Right panel: HD 19994 (solid line: $e=0.09$ ).

Echelle Spectrometer. In brief, we add a Keplerian signal to the existing velocity data, then attempt to recover that signal using a Lomb-Scargle periodogram. The mass of the simulated planet is increased until $99 \%$ of the injected signals are recovered with FAP $<0.1 \%$. For the constant stars in Wittenmyer et al. (2006), the null hypothesis is that no planets are present, and so the detection-limit algorithm can be applied directly to the velocity data. In the case of the known planet hosts, this null hypothesis no longer applies, and it would not do to "pre-whiten" those data by removing the known planet's orbit as if its parameters were known perfectly. The presence of an additional planet will act to modify the fitted parameters of the known planet. If two or more planets are present, and only one has been fitted, then part of the signal from the additional planets can be absorbed into the orbital elements of the one-planet fit. To approach this task with the maximum rigor, these effects must be accounted for. Hence, the detection-limit algorithm was modified in the following way: the test Keplerian signal was added to each of the original data sets, then these modified data sets were fitted for the known planet(s) using GaussFit. A residuals file was generated and then subjected to the periodogram search as described above. This process of fitting and removing the known planet occurred for every injected test signal. This method has the advantage of being essentially identical to the planet-search method described in Section 3.1.

\subsection{Results}

All data used in the fits for each planet host were subjected to the limit-determination routine, using 100 trial periods at even steps in the logarithm between 2 days and the total duration of observations. The results are plotted in Figures 17-27; planets with masses above the lines were recovered in $99 \%$ of trials (solid and dotted lines), or 50\% of trials (dashed lines), and 

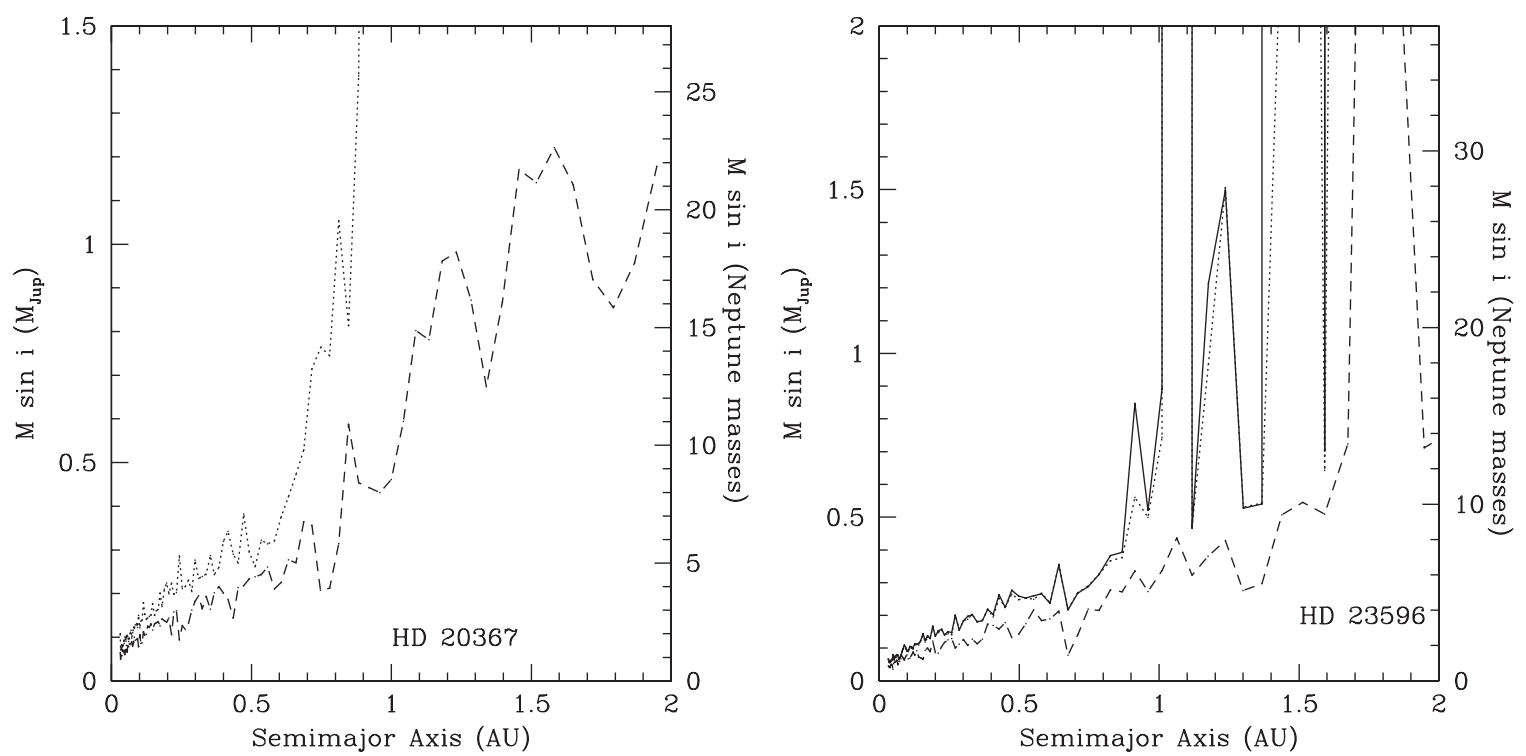

Figure 19. Left panel: same as Figure 17, but for HD 20367. These results were obtained without attempting to fit an existing planet, as no planet was confirmed in this system. Right panel: HD 23596 (solid line: $e=0.10$ ).
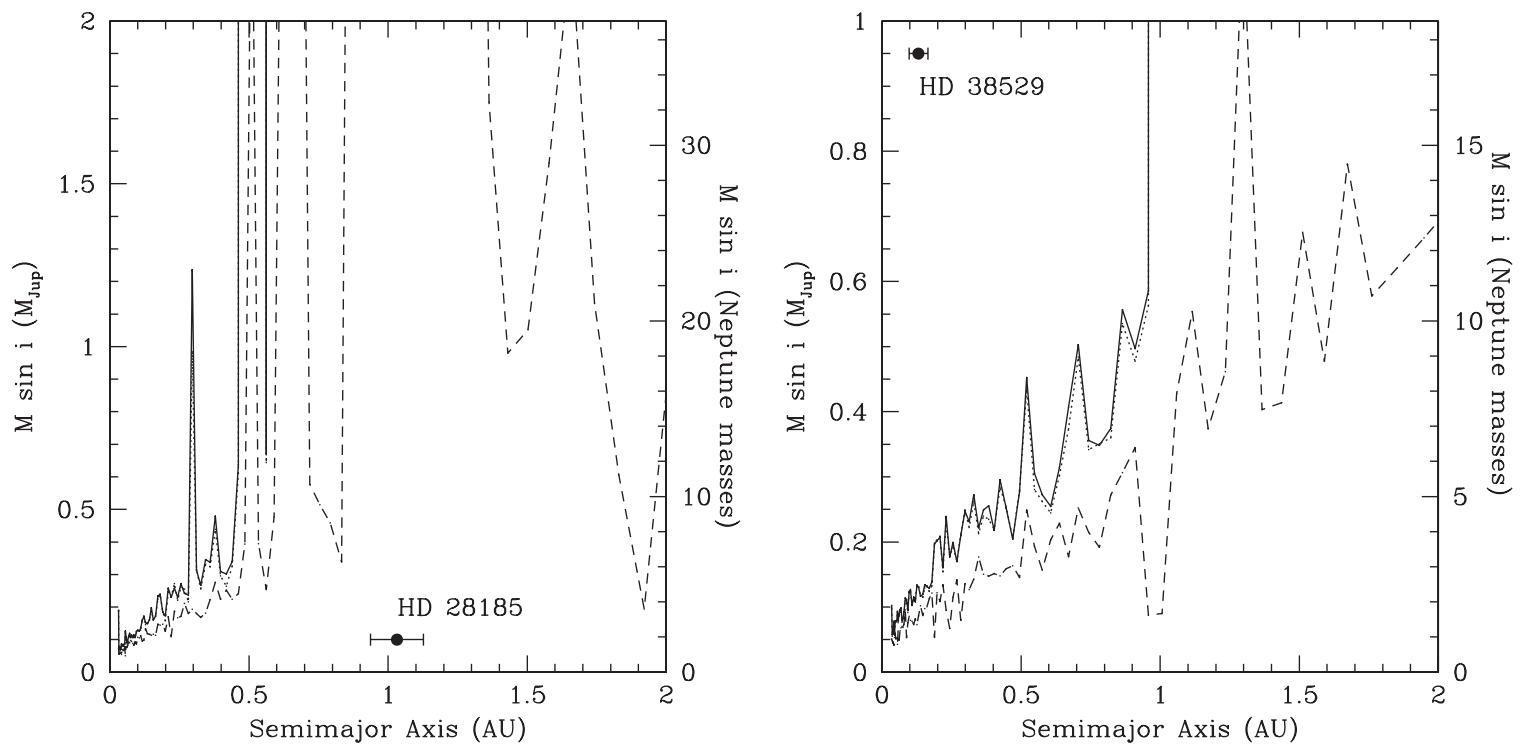

Figure 20. Left panel: same as Figure 17, but for HD 28185 (solid line: $e=0.09$ ). Right panel: HD 38529 (solid line: $e=0.12$ ).

hence can be ruled out by the data at those confidence levels, respectively. To match the parameter space specifically targeted in this study, and to match that of the test-particle simulations, the detection-limit plots show the inner $2 \mathrm{AU}$ only. For the eccentric trials (solid lines), the eccentricity of the injected test signals was chosen to be the mean eccentricity of the surviving test particles from the $\mathrm{N}$-body simulations described in Section 4.2. This approach was chosen because the dynamical simulations demonstrated that objects placed in circular orbits do not stay that way; the eccentricity of an undetected low-mass planet is expected to be influenced to nonzero values by the known giant planet.

It is important to note that the limits presented here represent the companions that can be ruled out by the data with $99 \%$ confidence. Lower mass planets could have been detected in this survey, but not necessarily at all (or 99\% of all) possible configurations. It is likely that a particular combination of parameters for a simulated planet makes that signal fiendishly difficult to recover by this method, owing to the known planet's radial-velocity signal and the sampling of the data. This is particularly important for simulated eccentric planets, where the velocity signal becomes markedly nonsinusoidal. The 50\% limits are also shown to illustrate the effect of relaxing the recovery criteria in order to reduce the impact of especially unfortunate configurations.

Table 6 summarizes the results of the detection-limit computations. The mean detection limits shown in Table 6 show that we could have detected $99 \%$ of planets with $M \sin i \sim$ 1.6 Neptune masses at $0.05 \mathrm{AU}$, and $M \sin i \sim 2.4 \mathrm{Nep}$ tune masses at $0.1 \mathrm{AU}$. The tightest limits were obtained for HD 3651, HD 108874, and $47 \mathrm{UMa}$, in which we are able to rule out Neptune-mass planets within $0.1 \mathrm{AU}$ at the $99 \%$ level. For all of the systems, the limits shown in Figures 17-27 exhibit some "blind spots" evident where the periodogram method failed to recover the injected signals with FAP $<0.1 \%$. Typically this occurs at certain trial periods for which the phase 

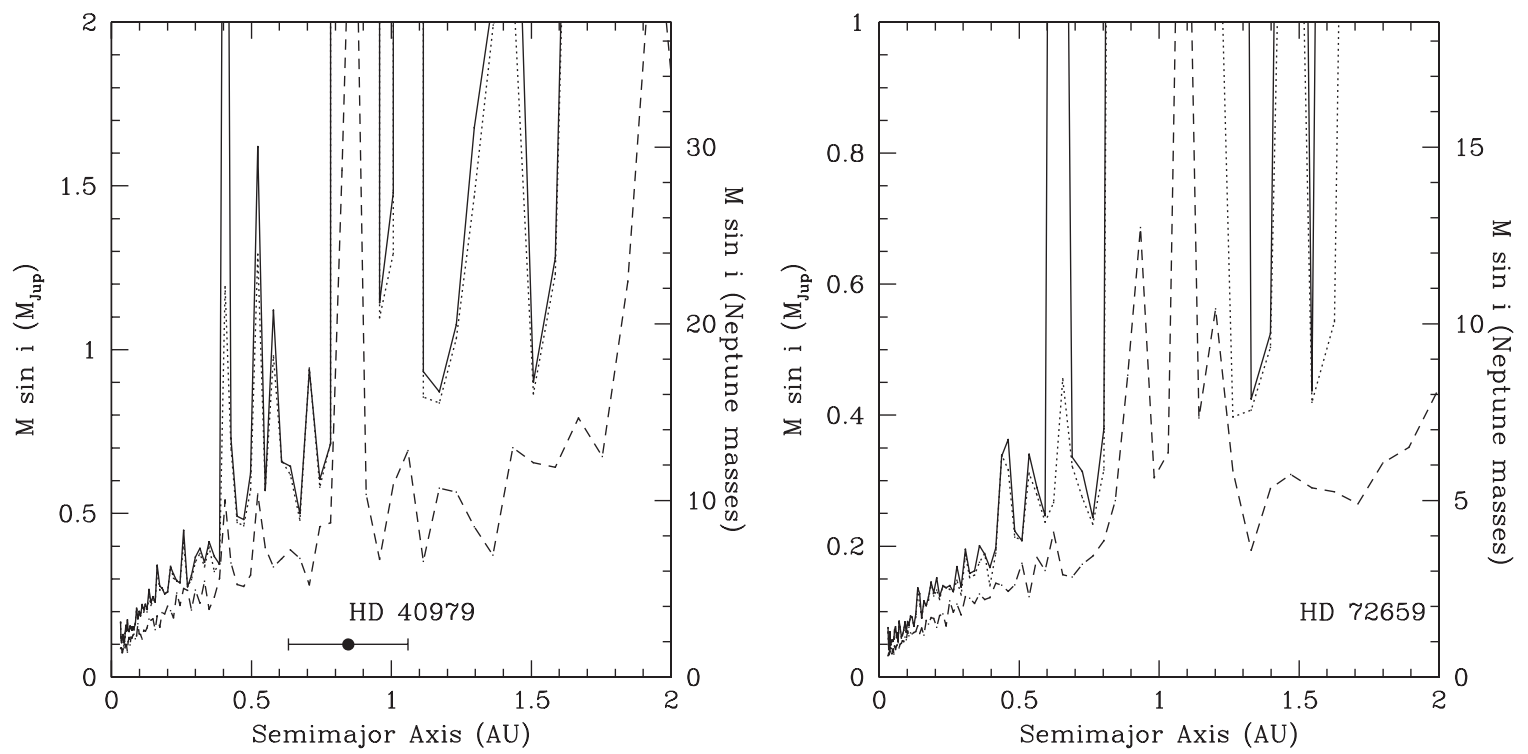

Figure 21. Left panel: same as Figure 17, but for HD 40979 (solid line: $e=0.11$ ). Right panel: HD 72659 (solid line: $e=0.10$ ).
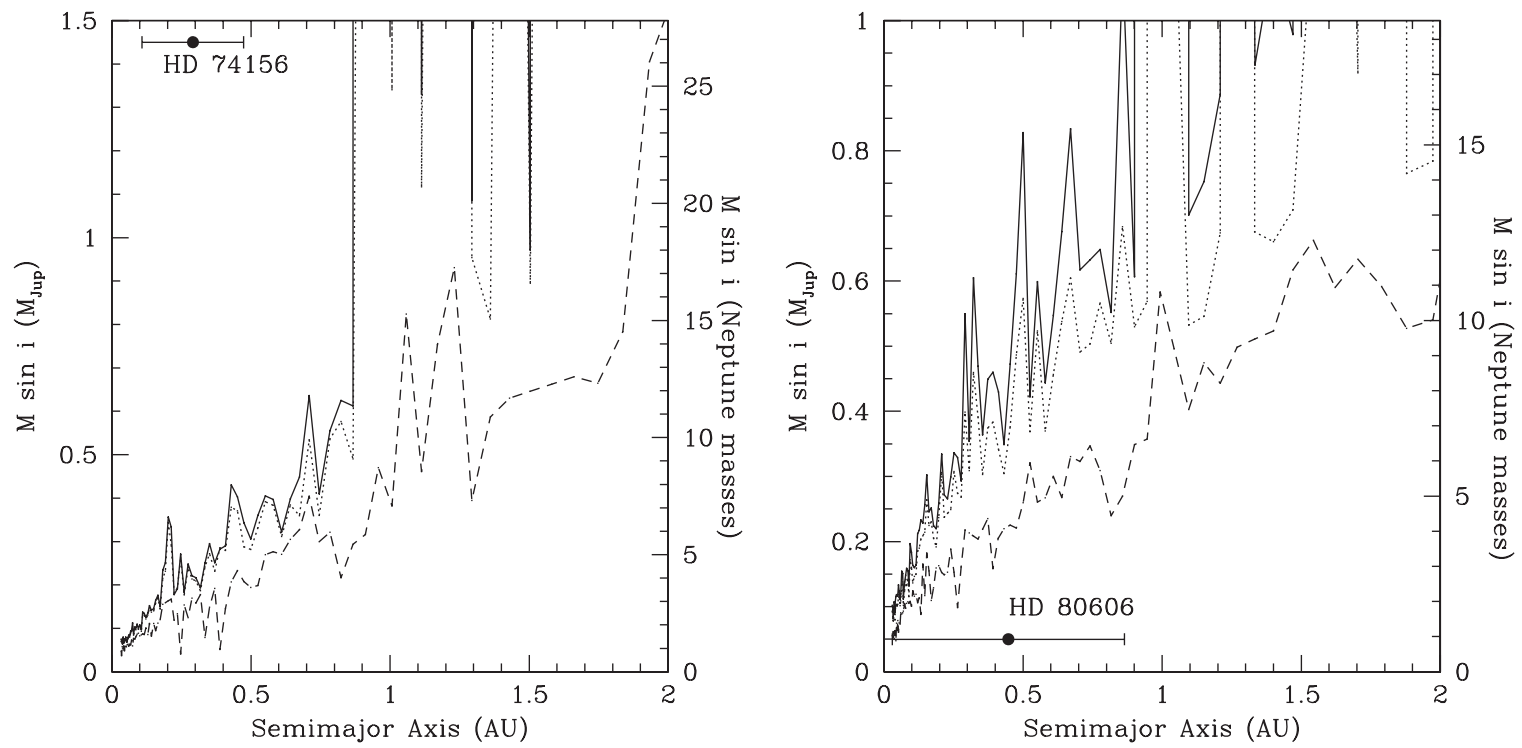

Figure 22. Left panel: same as Figure 17, but for HD 74156 (solid line: $e=0.15$ ). Right panel: HD 80606 (solid line: $e=0.31$ ).

coverage of the observational data is poor, and often at the 1month and 1-year windows. Using this method of fitting the known planet for each injected trial signal, such regions of ignorance are also present at periods close to that of the existing planet.

\section{DISCUSSION}

The aim of this project has been to intensively monitor known planetary systems in search of additional planets. However, in the sample of 22 planet hosts, the results have been quite the opposite. These new data cast doubt on the existence of two of the previously known planets, HD $20367 \mathrm{~b}$ and 47 UMa c (Wittenmyer et al. 2007a). The announcement by Bean et al. (2008) of a third planet in the HD 74156 system, one of the targets of this study, prompted a detailed investigation; at present we cannot confirm this object. These results suggest that systems with multiple giant planets are considerably more rare, or harder to detect, than anticipated at the outset of this project.
In this section, we will explore some reasons why no new multiple-planet systems were detected. Four possibilities are: (1) biases in the target selection conspire against detection of weak signals, (2) there exist fundamental physical differences between single- and multiple-planet systems, (3) we did not obtain a sufficient quantity of high-quality data, and (4) apparent single-planet systems may contain terrestrial-mass planets below the detection threshold.

\subsection{Biases in the Sample}

As with any scientific experiment, it is important to determine whether the sample selection resulted in unforeseen biases which affected the results. The target-selection process for this study, described in Section 2, included an intentional bias in favor of planet hosts with "large" (10-20 $\left.\mathrm{m} \mathrm{s}^{-1}\right)$ radial-velocity scatter about the orbital solution. The reasoning for this choice is straightforward: if a single planet can be fitted with minimal scatter, there is little room for additional undetected planets to hide in the residuals. An unintended consequence of this 

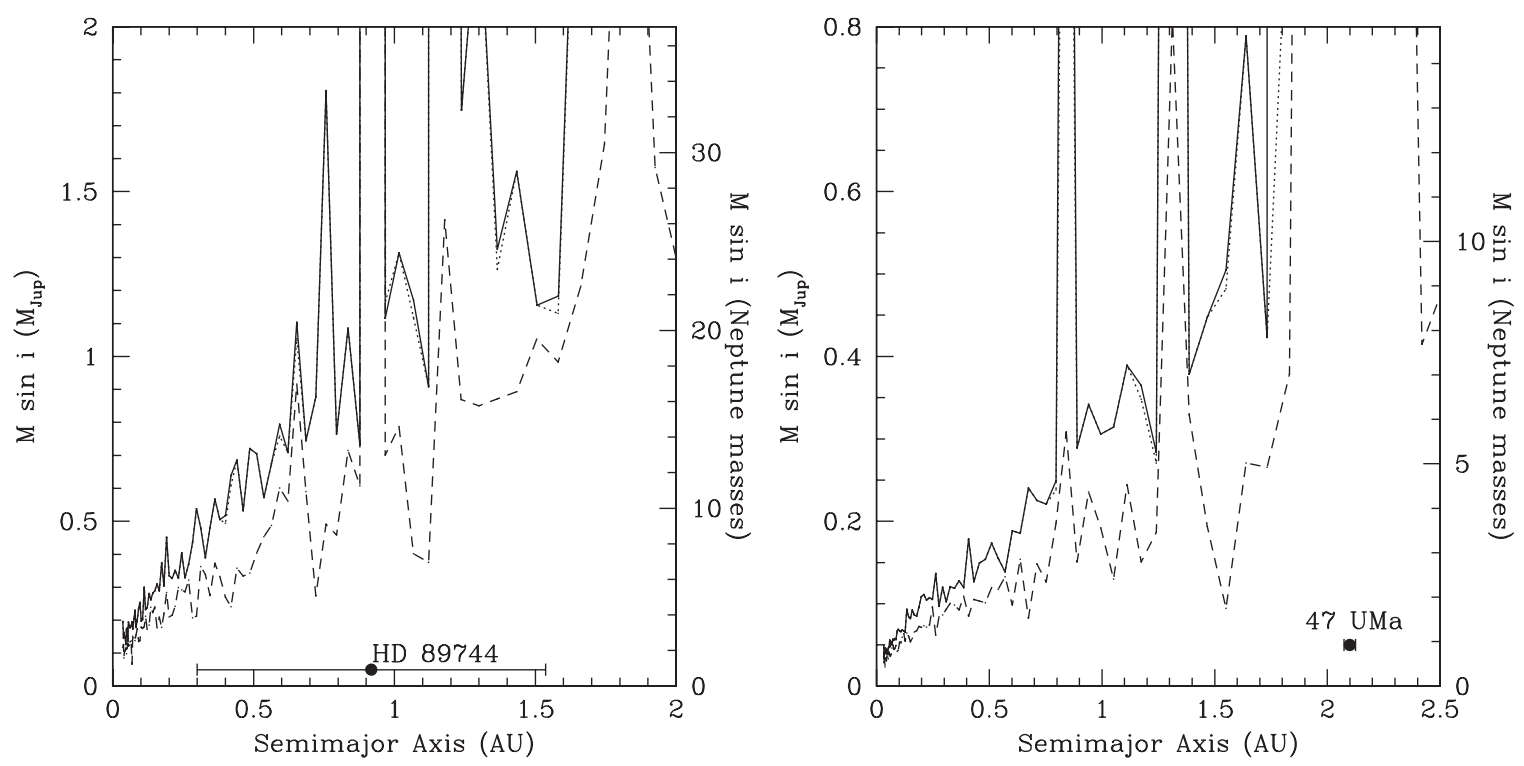

Figure 23. Left panel: same as Figure 17, but for HD 89744 (solid line: $e=0.01$ ). Right panel: $47 \mathrm{UMa}$ (solid line: $e=0.02$ ). Only $47 \mathrm{UMa} \mathrm{b}$ was included in the limit computations.
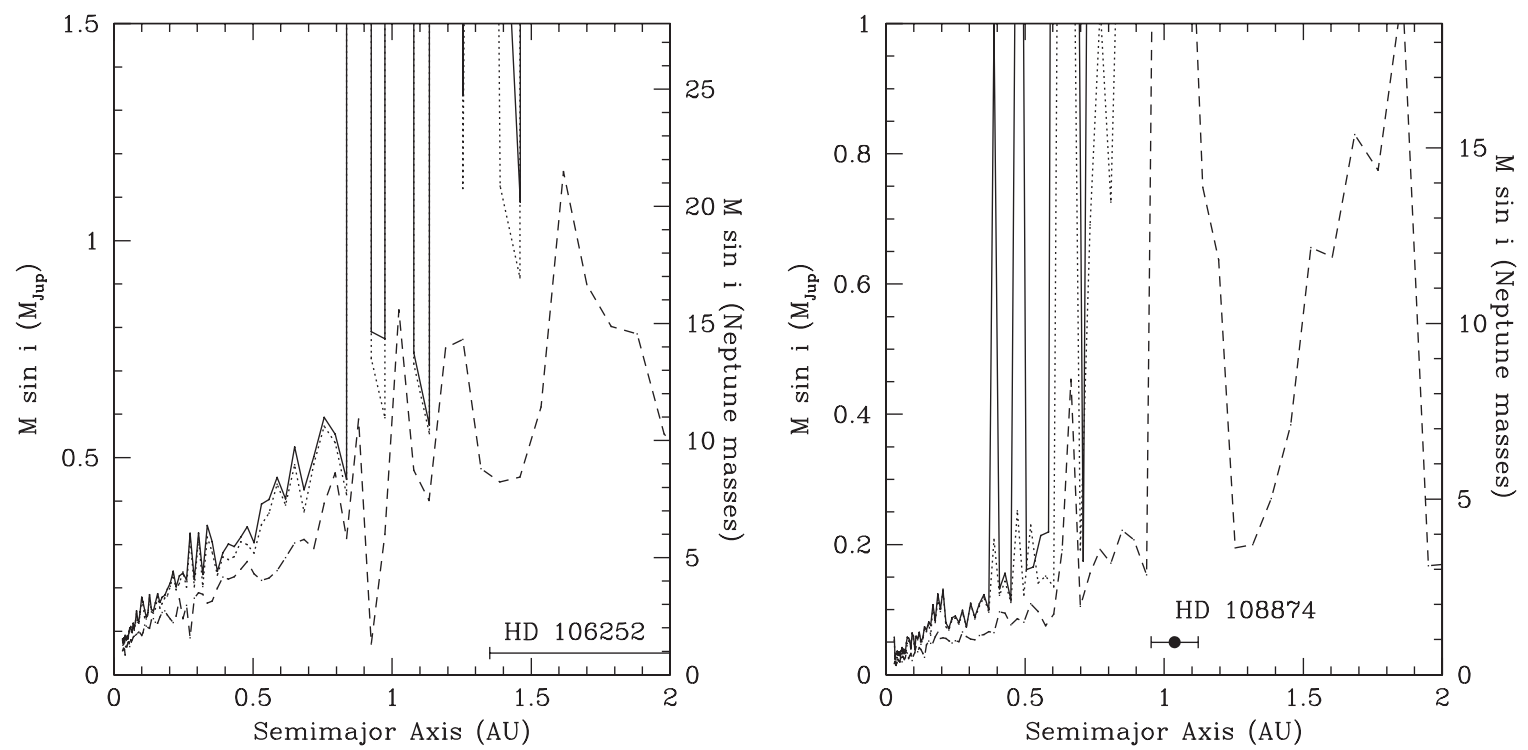

Figure 24. Left panel: same as Figure 17, but for HD 106252 (solid line: $e=0.15$ ). Right panel: HD 108874 (solid line: $e=0.15$ ).

selection criterion is that the excess scatter may be intrinsic to the star rather than indicative of additional planets. The achievable velocity precision improves with the number and strength of photospheric lines (Butler et al. 1996). Stars with higher temperatures or lower metallicities would have fewer and weaker lines, and result in lower velocity precision. In rapidly rotating stars, the spectral lines are broadened, which also degrades the radial-velocity precision. Fischer \& Valenti (2005) showed that the probability of a given star hosting a planet is positively correlated with its metallicity. In addition, those authors suggested that among planet host stars, metal-rich stars are more likely to host multiple planets. To check for these sorts of biases, we can perform a Kolmogorov-Smirnov (K-S) test to determine the probability that two samples are drawn from the same distribution. Comparing our sample of 22 planet host stars with other planet hosts not targeted $(N=200)$, the $\mathrm{K}-\mathrm{S}$ test shows no significant differences in $T_{\mathrm{eff}}(P=0.698)$, $[\mathrm{Fe} / \mathrm{H}](P=0.841)$, or $V \sin i(P=0.323)$. A comparison of the mean and median values of these quantities is shown in Table 7. The uncertainties are too large to make statistically meaningful comparisons, but the K-S test results suggest that there are no significant differences between the 22 planet hosts targeted here and those planet hosts not chosen.

\subsection{Fundamental Differences}

In this section, we ask the question, "Is there something special about the multi-planet systems"? Physical differences between single- and multiple-planet systems could arise either from the host star or from the processes of formation and dynamical evolution. Table 8 presents statistics on the planetary and stellar parameters for single- and multiple-planet systems. Only those planets detected by radial-velocity with $M \sin i<13$ $M_{\text {Jup }}$ were considered in the compilation of these statistics. Table 9 shows the results of $\mathrm{K}-\mathrm{S}$ tests on the planetary and stellar characteristics listed in Table 8. None of the parameters tested 

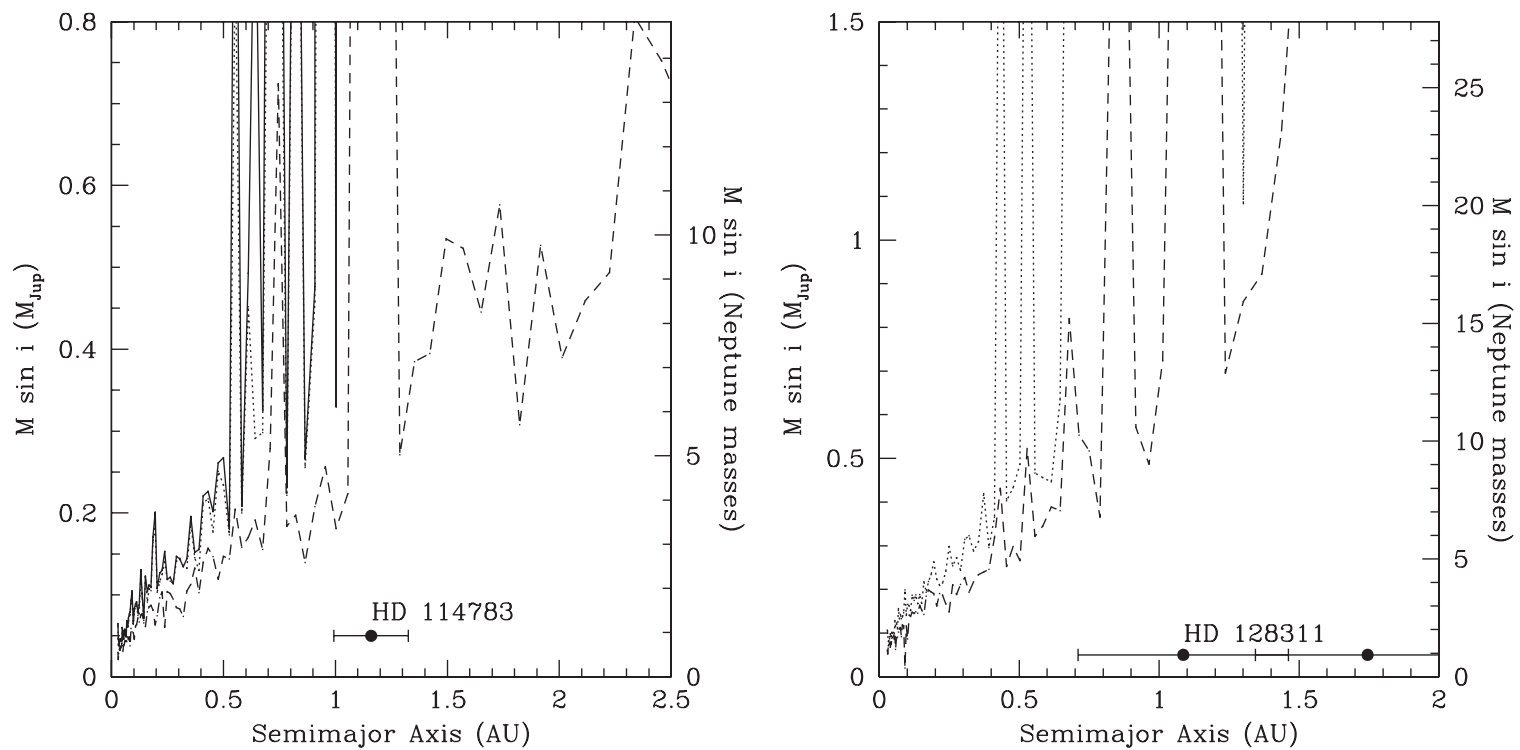

Figure 25. Left panel: same as Figure 17, but for HD 114783 (solid line: $e=0.11$ ). Right panel: HD 128311. Only circular orbits are considered since no test-particle simulations were conducted for this system.
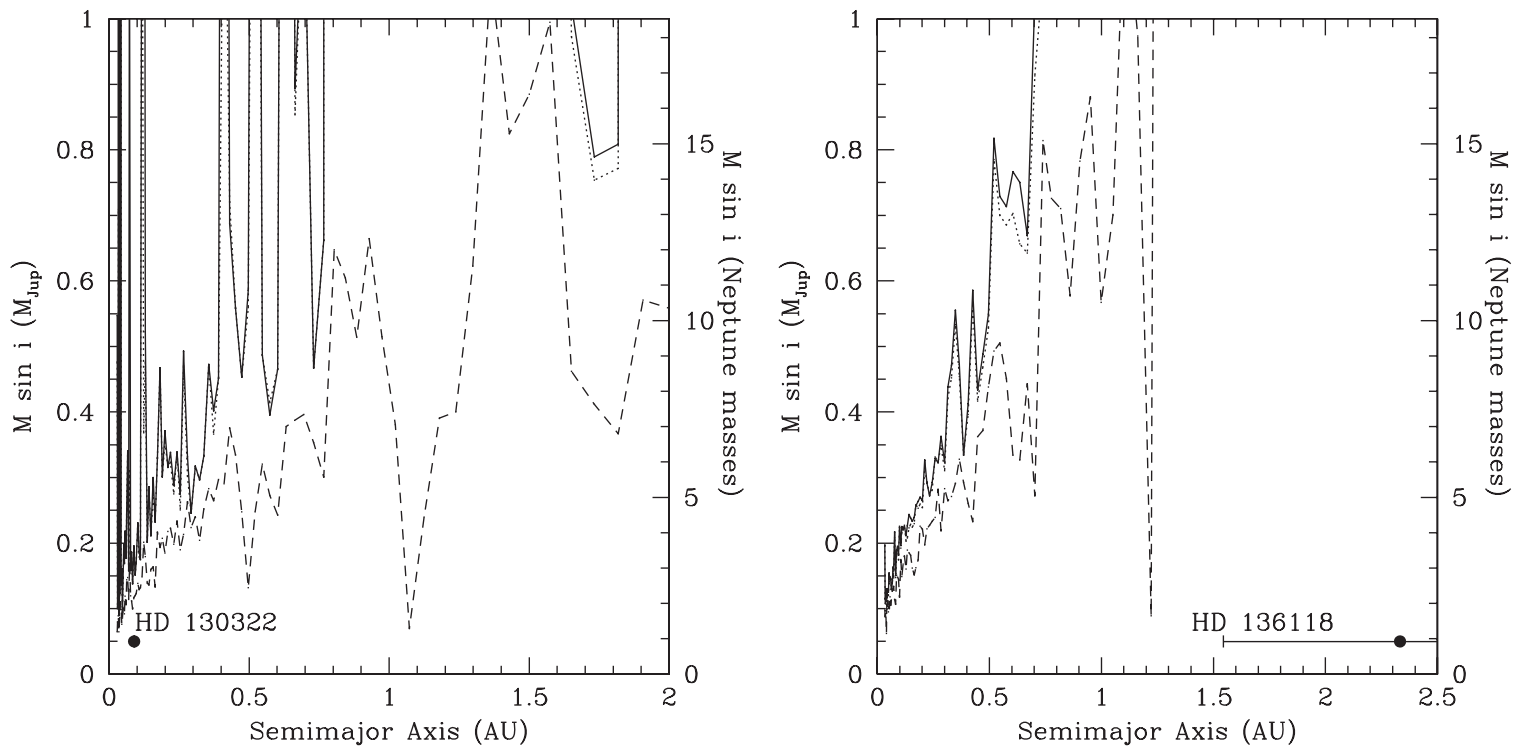

Figure 26. Left panel: same as Figure 17, but for HD 130322 (solid line: $e=0.02$ ). These results do not include data from Udry et al. (2000). Right panel: HD 136118 (solid line: $e=0.11$ ).

showed statistically significant differences between single- and multiple-planet systems. There are hints from the data in Table 8, and the $\mathrm{K}-\mathrm{S}$ test results in Table 9 that planets in multiple systems have larger $a$ and smaller $M \sin i$ than those in singleplanet systems. Both of these trends would work against the radial-velocity detection of planets in multiple systems. As the semimajor axis $a$ increases by a factor of $N$, the velocity semiamplitude $K$ decreases by $\sqrt{N}$, and as the planet mass decreases by a factor of $N, K$ also drops by a factor of $N$. It is also possible that a tendency toward lower mass and larger semimajor axis in multi-planet systems is the result of a selection effect. Once a single planet is found, follow-up observations may reveal longer period (larger $a$ ) planets, and intensive monitoring programs such as this work may then find lower mass planets. We can test whether a selection effect is at work by computing the statistics in Table 8 for the first planet discovered in the known multi-planet systems. These results are also given in Table 9; by comparing only the first planet found in the multiple systems with single planets, any significant difference between the distributions vanishes. Recently, Wright et al. (2009) have presented a detailed investigation of multipleplanet systems, and they find that planets in multiple systems tend to have lower eccentricities than single planets. We discuss this possibility in Section 6.5. Wright et al. (2008) also note that the orbital distances of planets in multiple systems are more evenly distributed in log-period, whereas single planets are more frequent at $a \sim 0.05 \mathrm{AU}$ and near $1 \mathrm{AU}$.

\subsection{Observing Strategy}

In considering whether there are important differences between the objects targeted in this work and known multi-planet systems, we can focus the comparison on the type of planetary system this survey was aimed at finding. The original motivation for this work was to investigate the possibility that systems 

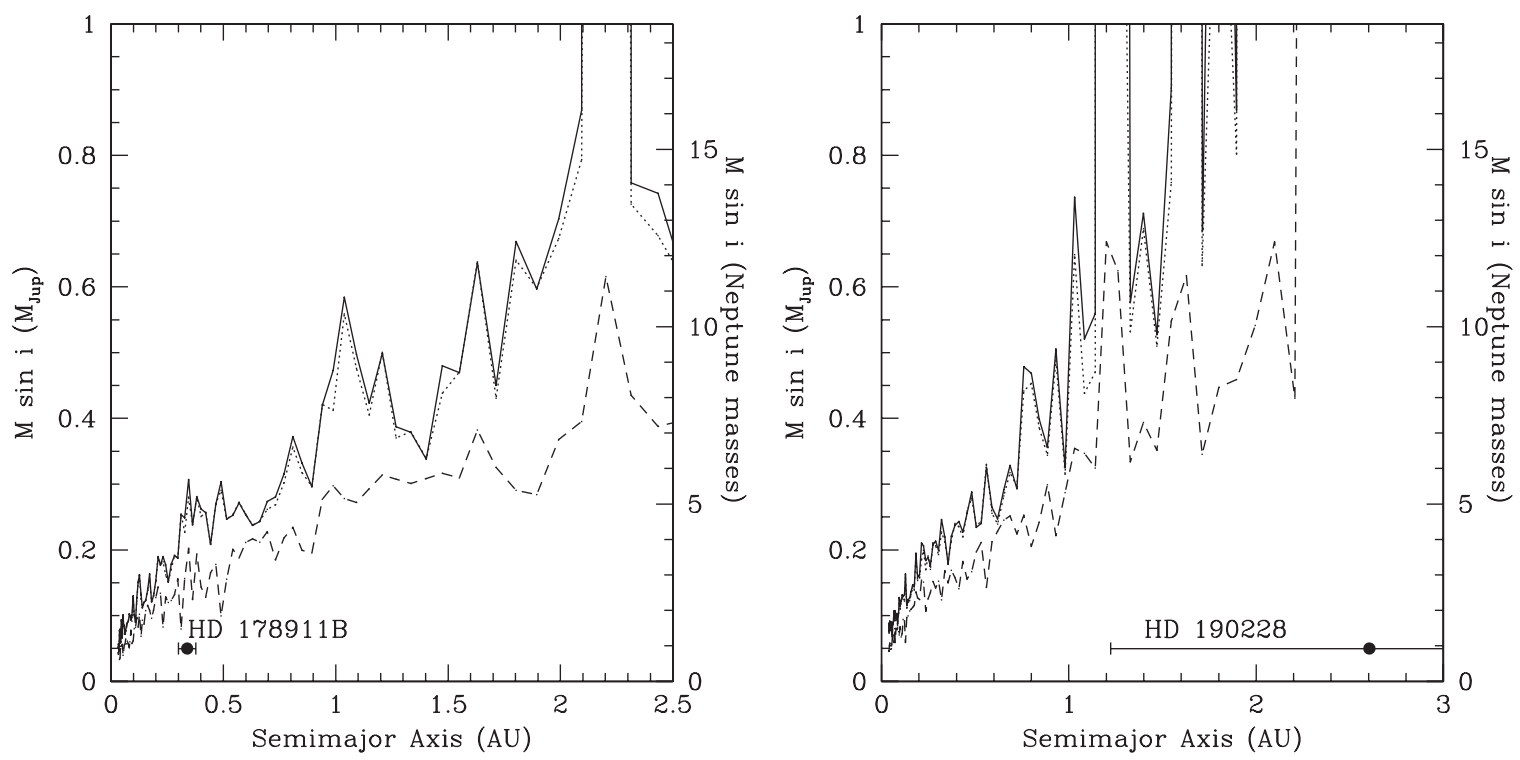

Figure 27. Left panel: same as Figure 17, but for HD 178911 B (solid line: $e=0.07$ ). Right panel: HD 190228 (solid line: $e=0.16$ ).

containing a Jovian planet also contain Neptune-mass planets ( 1 Neptune mass $\left.=0.054 M_{\text {Jup }}\right)$. At this writing, there are four such systems: 55 Cnc, GJ 876, $\mu$ Ara (= HD 160691), and GJ 777A (= HD 190360). With a sample size of only four, a meaningful statistical comparison of the host stars is not possible, but one can look at the characteristics of the body of radial-velocity data for these systems. In so doing, we ask whether those data are of exceptional quality or quantity which facilitated the detection of the additional low-mass planets in those systems. The recent detection of a fifth planet in the $55 \mathrm{Cnc}$ system by Fischer et al. (2008) used 636 measurements, binned into 250 Lick visits and 70 Keck visits. The detection of the fourth planet by McArthur et al. (2004) used 138 HET observations combined with 143 Lick data points (Marcy et al. 2002) and 48 data points from Naef et al. (2004). For $\mu$ Ara, the Neptune-mass planet was discovered using the HARPS spectrograph, which consistently delivers velocity precision of $\sim 1 \mathrm{~m} \mathrm{~s}^{-1}$ (Santos et al. 2004b; Pepe et al. 2007). The fourth planet in the $\mu$ Ara system (Pepe et al. 2007) was discovered using a total of 86 HARPS measurements combined with data from CORALIE and the AAT. The 18 $M_{\oplus}$ planet GJ 777Ac was discovered by Vogt et al. (2005) using 87 Keck velocities, and Rivera et al. (2005) found the 7.5 $M_{\oplus}$ GJ $876 \mathrm{~d}$ after 155 Keck observations. All four of these systems appear to have required an unusually large amount of the highest quality data from Keck and HARPS, with a mean of 107 data points. By contrast, the targets in this work each received an average of 53 HET visits. It is possible that the number of visits required to detect a hot Neptune was underestimated.

\subsection{Swarms of Earths}

Another possibility is that multiple-planet systems are indeed common, but, like our own solar system, contain many terrestrial-mass objects which are undetectable by current radial-velocity surveys. Core-accretion simulations by Ida \& Lin (2004a) predict a preponderance of $1-10 M_{\oplus}$ planets inside of $1 \mathrm{AU}$, and a "planet desert" in the range of 10-100 $M_{\oplus}$, arising due to rapid gas accretion by cores once they reach about $10 M_{\oplus}$. The current survey is not sensitive to the terrestrialmass objects, but planets within the "desert" could have been detected. Interestingly, Schlaufman et al. (2009) show that the presence of the planet desert could be confirmed by a radialvelocity survey with $1 \mathrm{~m} \mathrm{~s}^{-1}$ precision and $\sim 700$ observations, which is similar in scope to the present work. Of course, many more than 22 systems need to be studied before conclusions can be made, but the characterization of hundreds of new systems by the Kepler spacecraft (Borucki et al. 2003) will help to define the upper and lower mass boundaries of the planet desert. Ida \& Lin (2004a) note that the lower mass boundary would indicate the core mass required for rapid gas accretion, while the upper mass boundary would give insight into the mechanism by which gas accretion stops. Kepler discoveries of short-period super-Earths with masses $1-10 M_{\oplus}$ would lend further support to the core-accretion mechanism.

Simulations of planetesimal formation and migration also provide support for the existence of terrestrial-mass planets in systems with a gas giant planet. The GJ 876 system (Rivera et al. 2005), which contains two giant planets and an interior "super-Earth" ( $\left.M \sin i=7.5 M_{\oplus}\right)$, is thought to have originated by the shepherding of material as the giant planets migrated inward (Zhou et al. 2005). 200 Myr simulations by Raymond et al. (2006) and Mandell et al. (2007) resulted in the formation of planets with 1-5 Earth masses interior and exterior to the migrating hot Jupiter. Those models included only Type II migration, in which the migrating giant planet opens a gap in the protoplanetary disk. The models of Fogg \& Nelson (2007) consider the effects of Type I migration, in which the giant planet does not open a gap in the disk and inward drift is driven by differential torques on the planet. Inclusion of Type I migration did not alter the general outcome, that planets of several Earth masses are shepherded inward by the hot Jupiter, and some remain exterior to it. These models indicate that the inner regions of planetary systems may be populated with terrestrial-mass planets which would remain wholly undetectable by current radial-velocity surveys. Although this work achieved detection limits of 15-30 Earth masses, rocky planets in the range of 1-5 Earth masses could easily have been missed.

\subsection{Broader Implications for Planetary Systems}

We now take a step back and look at the bigger picture of planetary system formation and evolution. Based on the target 
Table 6

Companion Limit Summary

\begin{tabular}{|c|c|c|c|c|c|}
\hline Star & Eccentricity & $\begin{array}{c}M \sin i \\
0.05 \mathrm{AU} \\
\left(M_{\mathrm{Jup}}\right)\end{array}$ & $\begin{array}{l}M \sin i \\
0.1 \mathrm{AU} \\
\left(M_{\mathrm{Jup}}\right)\end{array}$ & $\begin{array}{c}\text { Median } K \\
99 \% \text { Recovery } \\
\left(\mathrm{m} \mathrm{s}^{-1}\right)\end{array}$ & $\begin{array}{c}\text { Median } K \\
\text { 50\% Recovery } \\
\left(\mathrm{m} \mathrm{s}^{-1}\right)\end{array}$ \\
\hline HD 3651 & 0.20 & 0.025 & 0.041 & 4.8 & $\ldots$ \\
\hline HD 3651 & 0 & 0.024 & 0.040 & 4.4 & 3.2 \\
\hline HD 8574 & 0.10 & 0.124 & 0.142 & 14.1 & $\ldots$ \\
\hline HD 8574 & 0 & 0.124 & 0.143 & 14.1 & 10.2 \\
\hline HD 10697 & 0.04 & 0.059 & 0.094 & 7.4 & $\ldots$ \\
\hline HD 10697 & 0 & 0.059 & 0.094 & 7.4 & 5.6 \\
\hline HD 19994 & 0.09 & 0.116 & 0.173 & 16.2 & $\ldots$ \\
\hline HD 19994 & 0 & 0.117 & 0.166 & 16.2 & 10.7 \\
\hline HD 20367 & 0 & 0.098 & 0.122 & 12.3 & 9.3 \\
\hline HD 23596 & 0.10 & 0.081 & 0.091 & 8.5 & $\ldots$ \\
\hline HD 23596 & 0 & 0.078 & 0.092 & 8.5 & 6.4 \\
\hline HD 28185 & 0.09 & 0.083 & 0.129 & 12.3 & $\ldots$ \\
\hline HD 38529 & 0.12 & 0.078 & 0.123 & 8.9 & $\ldots$ \\
\hline HD 38529 & 0 & 0.075 & 0.124 & 8.9 & 5.8 \\
\hline HD 40979 & 0.11 & 0.135 & 0.201 & 17.8 & $\ldots$ \\
\hline HD 40979 & 0 & 0.123 & 0.193 & 17.0 & 12.3 \\
\hline HD 72659 & 0.10 & 0.057 & 0.085 & 8.1 & $\ldots$ \\
\hline HD 72659 & 0 & 0.054 & 0.085 & 8.1 & 5.6 \\
\hline HD 74156 & 0.15 & 0.080 & 0.109 & 10.7 & $\ldots$ \\
\hline HD 74156 & 0 & 0.074 & 0.105 & 10.2 & 7.4 \\
\hline HD 80606 & 0.31 & 0.119 & 0.184 & 18.7 & $\ldots$ \\
\hline HD 80606 & 0 & 0.104 & 0.160 & 15.5 & 10.2 \\
\hline HD 89744 & 0.01 & 0.176 & 0.197 & 18.7 & $\ldots$ \\
\hline HD 89744 & 0 & 0.168 & 0.197 & 18.7 & 12.9 \\
\hline $47 \mathrm{UMa}$ & 0.02 & 0.039 & 0.067 & 6.1 & $\ldots$ \\
\hline $47 \mathrm{UMa}$ & 0 & 0.039 & 0.067 & 6.1 & 4.6 \\
\hline HD 106252 & 0.15 & 0.091 & 0.179 & 12.9 & $\ldots$ \\
\hline HD 108874 & 0 & 0.034 & 0.055 & 5.6 & 3.5 \\
\hline HD 114783 & 0.11 & 0.056 & 0.083 & 8.5 & $\ldots$ \\
\hline HD 114783 & 0 & 0.056 & 0.080 & 8.1 & 6.1 \\
\hline HD 128311 & 0 & 0.102 & 0.166 & 16.2 & 12.9 \\
\hline HD 130322 & 0.02 & 0.147 & 0.231 & 22.5 & $\ldots$ \\
\hline HD 130322 & 0 & 0.147 & 0.231 & 22.5 & 13.5 \\
\hline HD 136118 & 0.11 & 0.125 & 0.224 & 16.2 & $\ldots$ \\
\hline HD 136118 & 0 & 0.120 & 0.226 & 16.2 & 12.9 \\
\hline HD $178911 \mathrm{~B}$ & 0.07 & 0.061 & 0.130 & 10.2 & $\ldots$ \\
\hline HD $178911 \mathrm{~B}$ & 0 & 0.066 & 0.124 & 9.7 & 7.0 \\
\hline HD 190228 & 0.16 & 0.080 & 0.114 & 8.1 & $\ldots$ \\
\hline HD 190228 & 0 & 0.077 & 0.110 & 7.7 & 5.8 \\
\hline Mean (99\% recovery) & 0 & $0.087 \pm 0.036$ & $0.131 \pm 0.052$ & & \\
\hline Mean (50\% recovery) & 0 & $0.063 \pm 0.027$ & $0.090 \pm 0.036$ & & \\
\hline
\end{tabular}

selection and the resulting detection limits, this survey was most sensitive to systems with two giant planets (larger than Saturn mass). More specifically, our "key demographic" is a system with a "cold" Jupiter $(a \sim 1 \mathrm{AU})$ and a close-in planet with $M \sin i \gtrsim 1-2$ Neptune masses $\left(0.05-0.1 M_{\text {Jup }}\right)$. The detection limits given in Section 5 exclude such configurations at the $99 \%$ level for all of the planetary systems considered here. Systems containing a long-period, massive planet could also have been detected by trends or curvature in the velocity residuals; no such trends were present for any of the targets. This survey was much less sensitive to planetary systems like our own, with multiple terrestrial-mass planets and long-period giants, for the reasons discussed in Section 6.4. Planetary systems with architectures like our own solar system may yet be common, but we will need to wait for the results from Kepler to begin making quantitative statements.

The results of this work are most useful in assessing the frequency of planetary systems in which extensive migration has occurred, to bring two gas giant planets interior to the "snow line." In the core-accretion theory of giant planet formation (Pollack et al. 1996; Lissauer 1995), surface-density enhancement by ices facilitates the formation of $\sim 10-15 M_{\oplus}$ cores. The snow line, beyond which ices are present in the protoplanetary disk, has been estimated to lie at 1.6-1.8 AU in a minimum-mass solar nebula (Lecar et al. 2006). Perhaps the extensive migration required to construct systems with multiple giant planets with $a \lesssim 2 \mathrm{AU}$ is uncommon; the typical timescale in which a system is undergoing migration may be short. In other words, migration 
Table 7

Comparison of Stellar Characteristics

\begin{tabular}{lccc}
\hline \hline Quantity & Targets & Nontargets & Units \\
\hline$[\mathrm{Fe} / \mathrm{H}]$ (mean) & $0.12 \pm 0.18$ & $0.07 \pm 0.23$ & $\mathrm{dex}$ \\
{$[\mathrm{Fe} / \mathrm{H}]$ (median) } & 0.13 & 0.14 & $\mathrm{dex}$ \\
$T_{\text {eff }}($ mean) & $5741 \pm 361$ & $5608 \pm 496$ & $\mathrm{~K}$ \\
$T_{\text {eff }}($ median) & 5697 & 5704 & $\mathrm{~K}$ \\
$(B-V)$ (mean) & $0.67 \pm 0.11$ & $0.74 \pm 0.20$ & $\mathrm{mag}$ \\
$(B-V)$ (median) & 0.63 & 0.69 & $\mathrm{mag}$ \\
$V \sin i$ (mean) & $3.72 \pm 2.50$ & $2.75 \pm 1.72$ & $\mathrm{~km} \mathrm{~s}^{-1}$ \\
$V \sin i$ (median) & 2.48 & 2.40 & $\mathrm{~km} \mathrm{~s}^{-1}$ \\
\hline
\end{tabular}

Table 8

Characteristics of Single- and Multiple-Planet Systems

\begin{tabular}{lccc}
\hline \hline Quantity & Single & Multiple & Units \\
\hline$a$ (mean) & $0.95 \pm 1.05$ & $1.19 \pm 1.38$ & $\mathrm{AU}$ \\
$a$ (median) & 0.49 & 0.63 & $\mathrm{AU}$ \\
$e$ (mean) & $0.24 \pm 0.23$ & $0.19 \pm 0.17$ & \\
$e$ (median) & 0.18 & 0.16 & \\
$M$ sin $i$ (mean) & $2.72 \pm 3.16$ & $1.93 \pm 2.38$ & $M_{\text {Jup }}$ \\
$M$ sin $i$ (median) & 1.60 & 1.03 & $M_{\text {Jup }}$ \\
Star mass (mean) & $1.14 \pm 0.41$ & $1.06 \pm 0.32$ & $M_{\odot}$ \\
Star mass (median) & 1.07 & 1.04 & $M_{\odot}$ \\
{$[$ Fe $/ \mathrm{H}]$ (mean) } & $0.09 \pm 0.21$ & $0.05 \pm 0.30$ & $\mathrm{dex}$ \\
{$[$ Fe $/ \mathrm{H}]$ (median) } & 0.14 & 0.14 & $\mathrm{dex}$ \\
$T_{\text {eff }}$ (mean) & $5640 \pm 473$ & $5532 \pm 529$ & $\mathrm{~K}$ \\
$T_{\text {eff }}($ median) & 5724 & 5584 & $\mathrm{~K}$ \\
$(B-V)$ (mean) & $0.73 \pm 0.18$ & $0.77 \pm 0.22$ & $\mathrm{mag}$ \\
$(B-V)$ (median) & 0.68 & 0.72 & $\mathrm{mag}$ \\
\hline
\end{tabular}

may be fast, a hypothesis which has led to theoretical scenarios in which the observed planets are the last of many "batches" of planets which migrated onto the host star (Trilling et al. 2002; Ida \& Lin 2004a; Narayan et al. 2005). Type I migration, in which a net viscous torque on the protoplanet changes its orbit (Ward 1997), results in very fast migration with a timescale proportional to $M_{\text {planet }}^{-1}$. When a planet is massive enough $(0.3-$ $1.0 M_{\text {Jup }}$; Armitage 2007) to clear a gap in the disk, the slower Type II migration begins. The results of this work, showing a deficit of systems with multiple giant planets inside of 2-3 AU, suggest that they are dominated by Type I migration and rapidly accrete onto the star. Tanaka et al. (2002) showed that the Type I migration timescale is inversely proportional to the disk mass: planets in more massive disks migrate faster. If we make the reasonable assumption that multiple giant planets form from unusually massive disks, then Type I migration works against these planets surviving the migration if they remain below the gap-opening mass. To generate systems with multiple giant planets inside of 2-3 AU, migration should then be rapid enough to bring them there, but not so fast as to send the planets into the star. The results presented here suggest that such a scenario is uncommon.

In addition to migration, the dynamical history of planetary systems is an important factor in producing the observed architectures. The eccentricity distribution of extrasolar planets suggests that dynamically active histories are common. Interactions between giant planets can result in the ejection of one while imparting a significant eccentricity on the remaining planet (Rasio \& Ford 1996; Ford et al. 2005; Malmberg \& Davies 2009). Systems containing a single giant planet on a moderately eccentric orbit may be the result of such encounters, and thus less likely to host the sort of planets this survey was seeking.
Table 9

K-S Tests on Single- and Multiple-Planet Systems

\begin{tabular}{lc}
\hline \hline Quantity & K-S Probability \\
\hline$a$ & 0.004 \\
$a$ (first planet) & 0.249 \\
$M \sin i$ & 0.015 \\
$M \sin i$ (first planet) & 0.349 \\
$e$ & 0.125 \\
Star mass & 0.644 \\
{$[\mathrm{Fe} / \mathrm{H}]$} & 0.841 \\
$T_{\text {eff }}$ & 0.135 \\
$(B-V)$ & 0.383 \\
\hline
\end{tabular}

Note. ${ }^{\text {a }}$ Probability that the two samples are drawn from the same distribution.

Table 10

Radial-Velocity Data

\begin{tabular}{lcc}
\hline \hline JD-2400000 & Velocity $\left(\mathrm{m} \mathrm{s}^{-1}\right)$ & Uncertainty $\left(\mathrm{m} \mathrm{s}^{-1}\right)$ \\
\hline 53581.21162 & 14.4 & 3.7 \\
53600.79860 & 0.4 & 4.7 \\
53604.79357 & -6.7 & 3.5 \\
53606.78360 & -9.3 & 3.9 \\
53608.77426 & -10.9 & 3.6 \\
53615.96471 & -18.5 & 3.6 \\
53628.74240 & 3.8 & 3.3 \\
53669.61203 & -12.2 & 3.6 \\
53678.79142 & -10.0 & 3.6 \\
53682.78611 & -18.1 & 3.3 \\
53687.77875 & 17.0 & 3.7 \\
53691.76158 & 12.3 & 3.9 \\
53694.75466 & 16.7 & 3.7 \\
53696.76029 & 15.5 & 3.4 \\
53955.83593 & 12.3 & 3.8 \\
53956.83044 & 7.3 & 3.9 \\
53957.82392 & 10.6 & 3.5 \\
53973.80980 & -4.3 & 4.7 \\
53976.78586 & -13.2 & 3.4 \\
53978.97197 & -2.2 & 5.9 \\
53985.95982 & -15.2 & 4.4 \\
53987.95527 & -13.6 & 3.0 \\
53989.74009 & -20.6 & 2.8 \\
54003.70817 & 13.2 & 4.4 \\
54005.68492 & 17.2 & 3.6 \\
54056.78111 & -9.9 & 3.5 \\
54062.55312 & 19.0 & 3.4 \\
54064.54902 & 13.0 & 3.3 \\
54130.55508 & 15.4 & 3.4 \\
54282.92879 & -1.7 & 4.4 \\
54352.96182 & -9.8 & 3.1 \\
54394.64607 & -1.9 & 3.5 \\
54399.61380 & -9.2 & 3.6 \\
54414.77832 & -1.0 & 4.4 \\
54423.75714 & -14.9 & \\
\hline & & \\
& & \\
& &
\end{tabular}

Notes. This table is available in its entirety in a machine-readable form in the online journal. A portion is shown here for guidance regarding its form and content.

The median eccentricity of the planets targeted in this work is 0.29 , compared to a median $e$ of 0.15 for all other planets. Comparing the distributions by the $\mathrm{K}-\mathrm{S}$ test gives a probability of 0.048 , indicating a marginally significant difference between the two. Fischer et al. (2008) use the relatively low eccentricities $(e<0.2)$ of the five $55 \mathrm{Cnc}$ planets to suggest that a benign dynamical history allowed so many planets to remain. The GJ 876, HD 37124, HD 73526, and GJ 581 systems also have 
multiple planets with $e<0.2$, but counterexamples are found in HD 160691, HD 74156, and HD $202206\left(e_{\max }=0.57,0.64\right.$, and 0.44 , respectively). An uneventful dynamical history contributes to a planetary system's observed end state, but comprises only a part of the picture in combination with its formation history.

A primary goal of the search for extrasolar planets is to estimate how common the architecture of our own solar system might be. If the processes of planet formation and migration form many systems similar to our own, it becomes more likely that Earth-like planets may be present. The results of this work indicate that planetary systems like our own may be common if (1) terrestrial-mass planets are present but undetected, or (2) Type I migration timescales are so short that multiple giant planets rarely end up within 2-3 AU. Conversely, our solar system may be rare if the dynamical history of most planetary systems results in many ejections and high eccentricities.

\section{SUMMARY}

We have carried out an intensive radial-velocity campaign to monitor 22 known planetary systems for additional planets. No new planets were found, and these new data do not support the proposed planets HD 20367 b, HD 74156 d, and 47 UMa c. We have used test particles and Saturn-mass bodies to probe 20 planetary systems for regions in which additional planets could exist. The massive-body results are consistent with the testparticle results: each of these systems has regions, sometimes quite large, where additional planets may remain in stable orbits. Finally, we show that this campaign could have detected $99 \%$ of planets with $M \sin i \lesssim 2.6$ Neptune masses within $0.10 \mathrm{AU}$.

This material is based on work supported by the $\mathrm{Na}$ tional Aeronautics and Space Administration under grants NNG04G141G, NNG05G107G issued through the Terrestrial Planet Finder Foundation Science program and grant NNX07AL70G issued through the Origins of Solar Systems Program. We are grateful to the HET TAC for their generous allocation of telescope time for this project. Much of the computing for the dynamical simulations used the Lonestar cluster at the Texas Advanced Computing Center. This research has made use of NASA's Astrophysics Data System (ADS) and the SIMBAD database, operated at CDS, Strasbourg, France. The Hobby-Eberly Telescope (HET) is a joint project of the University of Texas at Austin, the Pennsylvania State University, Stanford University, Ludwig-Maximilians-Universität München, and Georg-August-Universität Göttingen. The HET is named in honor of its principal benefactors, William P. Hobby and Robert E. Eberly.

\section{REFERENCES}

Baranne, A., et al. 1996, A\&AS, 119, 373

Barnes, R., Goździewski, K., \& Raymond, S. N. 2008, ApJ, 680, L57

Barnes, R., \& Quinn, T. 2004, ApJ, 611, 494

Barnes, R., \& Raymond, S. N. 2004, ApJ, 617, 569

Bean, J. L., McArthur, B. E., Benedict, G. F., \& Armstrong, A. 2008, ApJ, 672 1202

Bean, J. L., McArthur, B. E., Benedict, G. F., Harrison, T. E., Bizyaev, D., Nelan, E., \& Smith, V. V. 2007, AJ, 134, 749

Bodenheimer, P., Hubickyj, O., \& Lissauer, J. J. 2000, Icarus, 143, 2

Bonfils, X., et al. 2007, A\&A, 474, 293

Borucki, W. J., et al. 2003, Proc. SPIE, 4854, 129

Butler, R. P., Marcy, G. W., Vogt, S. S., Fischer, D. A., Henry, G. W., Laughlin, G., \& Wright, J. T. 2003, ApJ, 582, 455

Boss, A. P. 1995, Science, 267, 360

Boss, A. P. 1998, ApJ, 503, 923
Boss, A. P. 2003, ApJ, 599, 577

Bouchy, F., et al. 2009, A\&A, 496, 527

Butler, R. P., Marcy, G. W., Williams, E., McCarthy, C., Dosanjh, P., \& Vogt, S. S. 1996, PASP, 108, 500

Butler, R. P., et al. 2006, ApJ, 646, 505

Chambers, J. E. 1999, MNRAS, 304, 793

Chatterjee, S., Ford, E. B., Matsumura, S., \& Rasio, F. A. 2008, ApJ, 686, 580 Cochran, W. D., et al. 2004, ApJ, 611, L133

Endl, M., Cochran, W. D., Wittenmyer, R. A., \& Boss, A. P. 2008, ApJ, 673 1165

Endl, M., Kürster, M., \& Els, S. 2000, A\&A, 362, 585

Endl, M., Kürster, M., Els, S. H. A. P., Cochran, W. D., Dennerl, K., \& Döbereiner, S. 2002, A\&A, 392, 671

Fischer, D. A., Marcy, G. W., Butler, R. P., Laughlin, G., \& Vogt, S. S. 2002, ApJ, 564, 1028

Fischer, D. A., \& Valenti, J. 2005, ApJ, 622, 1102

Fischer, D. A., et al. 2008, ApJ, 675, 790

Fogg, M. J., \& Nelson, R. P. 2007, A\&A, 472, 1003

Ford, E. B., Lystad, V., \& Rasio, F. A. 2005, Nature, 434, 873

Goździewski, K., \& Konacki, M. 2006, ApJ, 647, 573

Henry, G. W. 1999, PASP, 111, 845

Holmberg, J., Nordström, B., \& Andersen, J. 2007, A\&A, 475, 519

Horne, J. H., \& Baliunas, S. L. 1986, ApJ, 302, 757

Ida, S., \& Lin, D. N. C. 2004a, ApJ, 604, 388

Ida, S., \& Lin, D. N. C. 2004b, ApJ, 616, 567

Ida, S., \& Lin, D. N. C. 2008, ApJ, 673, 487

Jefferys, W. H., Fitzpatrick, M. J., \& McArthur, B. E. 1987, Celest. Mech., 41, 39

Jones, B. W., Sleep, P. N., \& Chambers, J. E. 2001, A\&A, 366, 254

Jurić, M., \& Tremaine, S. 2008, ApJ, 686, 603

Kürster, M., Schmitt, J. H. M. M., Cutispoto, G., \& Dennerl, K. 1997, A\&A, 320,831

Laughlin, G., \& Chambers, J. E. 2002, AJ, 124, 592

Lecar, M., Podolak, M., Sasselov, D., \& Chiang, E. 2006, ApJ, 640, 1115

Levison, H. F., \& Duncan, M. J. 1994, Icarus, 108, 18

Levison, H. F., Lissauer, J. J., \& Duncan, M. J. 1998, AJ, 116, 1998

Lissauer, J. J. 1995, Icarus, 114, 217

Lomb, N. R. 1976, Ap\&SS, 39, 447

Lovis, C., et al. 2006, Nature, 441, 305

Malmberg, D., \& Davies, M. B. 2009, MNRAS, 394, L26

Mandell, A. M., Raymond, S. N., \& Sigurdsson, S. 2007, ApJ, 660, 823

Marcy, G. W., \& Butler, R. P. 1992, PASP, 104, 270

Marcy, G. W., Butler, R. P., Fischer, D. A., Laughlin, G., Vogt, S. S., Henry, G. W., \& Pourbaix, D. 2002, ApJ, 581, 1375

Mayor, M., Udry, S., Naef, D., Pepe, F., Queloz, D., Santos, N. C., \& Burnet, M. 2004, A\&A, 415, 391

Mayor, M., et al. 2009, A\&A, 493, 639

McArthur, B. E., et al. 2004, ApJ, 614, L81

Menou, K., \& Tabachnik, S. 2003, ApJ, 583, 473

Naef, D., Mayor, M., Beuzit, J. L., Perrier, C., Queloz, D., Sivan, J. P., \& Udry, S. 2004, A\&A, 414, 351

Naef, D., et al. 2001, A\&A, 375, L27

Narayan, R., Cumming, A., \& Lin, D. N. C. 2005, ApJ, 620, 1002

Noyes, R. W., Hartmann, L. W., Baliunas, S. L., Duncan, D. K., \& Vaughan, A. H. 1984, ApJ, 279, 763

Paulson, D. B., Saar, S. H., Cochran, W. D., \& Hatzes, A. P. 2002, AJ, 124, 572 Pepe, F., et al. 2007, A\&A, 462, 769

Perrier, C., Sivan, J.-P., Naef, D., Beuzit, J. L., Mayor, M., Queloz, D., \& Udry, S. 2003, A\&A, 410, 1039

Pollack, J. B., Hubickyj, O., Bodenheimer, P., Lissauer, J. J., Podolak, M., \& Greenzweig, Y. 1996, Icarus, 124, 62

Ramsey, L. W., et al. 1998, Proc. SPIE, 3352, 34

Rasio, F. A., \& Ford, E. B. 1996, Science, 274, 954

Raymond, S. N., \& Barnes, R. 2005, ApJ, 619, 549

Raymond, S. N., Mandell, A. M., \& Sigurdsson, S. 2006, Science, 313, 1413

Rivera, E., \& Haghighipour, N. 2007, MNRAS, 374, 599

Rivera, E. J., \& Lissauer, J. J. 2000, ApJ, 530, 454

Rivera, E. J., \& Lissauer, J. J. 2001, ApJ, 558, 392

Rivera, E. J., et al. 2005, ApJ, 634, 625

Santos, N. C., Israelian, G., \& Mayor, M. 2004a, A\&A, 415, 1153

Santos, N. C., Mayor, M., Naef, D., Pepe, F., Queloz, D., Udry, S., \& Burnet, M. 2001, A\&A, 379, 999

Santos, N. C., et al. 2004b, A\&A, 426, L19

Scargle, J. D. 1982, ApJ, 263, 835

Schlaufman, K. C., Lin, D. N. C., \& Ida, S. 2009, ApJ, 691, 1322

Takeda, G., Ford, E. B., Sills, A., Rasio, F. A., Fischer, D. A., \& Valenti, J. A. 2007, ApJS, 168, 297 
Tanaka, H., Takeuchi, T., \& Ward, W. R. 2002, ApJ, 565, 1257

Trilling, D. E., Benz, W., Guillot, T., Lunine, J. I., Hubbard, W. B., \& Burrows, A. 1998, ApJ, 500, 428

Trilling, D. E., Lunine, J. I., \& Benz, W. 2002, A\&A, 394, 241

Tull, R. G. 1998, Proc. SPIE, 3355, 387

Udry, S., Mayor, M., \& Queloz, D. 2003, ASP Conf. 294, Sci. Frontiers Res. Extrasolar Planets, ed. D. Dewing \& S. Seager, 17

Udry, S., et al. 2000, A\&A, 356, 590

Udry, S., et al. 2007, A\&A, 469, L43

Valenti, J. A., \& Fischer, D. A. 2005, ApJS, 159, 141

Vogt, S. S., Butler, R. P., Marcy, G. W., Fischer, D. A., Henry, G. W., Laughlin, G., Wright, J. T., \& Johnson, J. A. 2005, ApJ, 632, 638

Vogt, S. S., Butler, R. P., Marcy, G. W., Fischer, D. A., Pourbaix, D., Apps, K., \& Laughlin, G. 2002, ApJ, 568, 352
Ward, W. R. 1997, Icarus, 126, 261

Wittenmyer, R. A., Endl, M., \& Cochran, W. D. 2007a, ApJ, 654, 625

Wittenmyer, R. A., Endl, M., Cochran, W. D., Hatzes, A. P., Walker, G. A. H., Yang, S. L. S., \& Paulson, D. B. 2006, AJ, 132, 177

Wittenmyer, R. A., Endl, M., Cochran, W. D., \& Levison, H. F. 2007b, AJ, 134, 1276

Wright, J. T., Marcy, G. W., Butler, R. P., \& Vogt, S. S. 2004, ApJS, 152, 261

Wright, J. T., Upadhyay, S., Marcy, G. W., Fischer, D. A., Ford, E. B., \& Johnson, J. A. 2009, ApJ, 693, 1084

Wright, J. T., et al. 2007, ApJ, 657, 533

Zhou, J.-L., Aarseth, S. J., Lin, D. N. C., \& Nagasawa, M. 2005, ApJ, 631, L85

Zucker, S., et al. 2002, ApJ, 568, 363 